\title{
Cesium Substitution Disrupts Concerted Cation Dynamics in Formamidinium Hybrid Perovskites
}

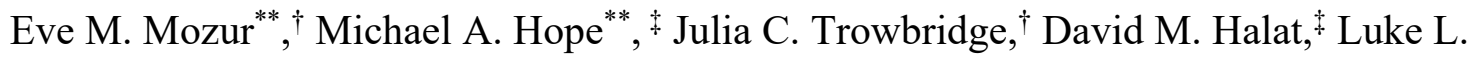 \\ Daemen, ${ }^{\natural}$ Annalise E. Maughan, ${ }^{\dagger}$ Timothy R. Prisk, ${ }^{\S}$ Clare P. Grey,${ }^{\ddagger}$ and James R. Neilson*† \\ 'Department of Chemistry, Colorado State University, Fort Collins, CO, 80523 \\ tDepartment of Chemistry, University of Cambridge, Lensfield Road, Cambridge, CB2 1EW, \\ U.K \\ 'Chemical and Engineering Materials Division, Oak Ridge National Laboratory, Oak Ridge, TN, \\ 37831, United States \\ ${ }^{\S}$ Center for Neutron Research National Institute of Standards and Technology, Gaithersburg, \\ MD, 20899, USA \\ *jrn@colostate.edu
}

\begin{abstract}
Although initial studies on hybrid perovskites for photovoltaic applications focused on simple compositions, the most technologically relevant perovskites are heavily substituted. The influence of chemical substitution on the general phase behavior and specific physical properties remains ambiguous. The hybrid perovskite formamidinium lead bromide, $\mathrm{CH}\left(\mathrm{NH}_{2}\right)_{2} \mathrm{PbBr}_{3}$, exhibits complex phase behavior manifesting in a series of crystallographically-unresolvable phase transitions associated with changes in the cation dynamics. Here, we characterize the molecular and lattice dynamics of $\mathrm{CH}\left(\mathrm{NH}_{2}\right)_{2} \mathrm{PbBr}_{3}$ as a function of temperature, and their evolution upon chemical substitution of $\mathrm{CH}\left(\mathrm{NH}_{2}\right)_{2}{ }^{+}$for cesium $\left(\mathrm{Cs}^{+}\right)$with crystallography, neutron scattering, ${ }^{1} \mathrm{H}$ and ${ }^{14} \mathrm{~N}$ nuclear magnetic resonance spectroscopy, and ${ }^{79} \mathrm{Br}$ nuclear quadrupolar spectroscopy. $\mathrm{Cs}^{+}$ substitution suppresses the four low-temperature phase transitions of $\mathrm{CH}\left(\mathrm{NH}_{2}\right)_{2} \mathrm{PbBr}_{3}$, which propagate through concerted changes in the dynamic degrees of freedom of the organic sub-lattice and local or long-range distortions of the octahedral framework. We propose that cesium substitution suppress the phase transitions through the relief of geometric frustration associated
\end{abstract}

** Both authors contributed equally. 
with the orientations of $\mathrm{CH}\left(\mathrm{NH}_{2}\right)_{2}{ }^{+}$molecules, which retain their local dynamical degrees of freedom.

\section{Introduction}

Hybrid halide perovskites tend to be more compositionally complex than compound inorganic semiconductors, especially as most hybrid perovskites in devices are highly substituted. For example, the unsubstituted hybrid perovskites $\mathrm{CH}_{3} \mathrm{NH}_{3} \mathrm{~Pb} X_{3}$ and $\mathrm{CH}\left(\mathrm{NH}_{2}\right)_{2} \mathrm{~Pb} X_{3}(X=\mathrm{I}, \mathrm{Br}, \mathrm{Cl})$, are not stable under ambient conditions, which has been attributed to their positive enthalpies of formation. ${ }^{1,2}$ Recent calculations suggest that $A$-site mixing can decrease the Gibbs free energy of a given hybrid perovskite, ${ }^{3}$ which aligns with studies showing that chemical substitution at the $A$ and $X$ sites prevents decomposition to the binaries or competing non-perovskite phases. ${ }^{4-7}$ While unsubstituted $\mathrm{CH}_{3} \mathrm{NH}_{3} \mathrm{PbI}_{3}$ decomposes into the binaries in 20 hours under $85 \%$ relative humidity, partial substitution of $\mathrm{CH}_{3} \mathrm{NH}_{3}{ }^{+}$with $\mathrm{CH}\left(\mathrm{NH}_{2}\right)_{2}{ }^{+}$prevents decomposition under the same conditions. ${ }^{8}$ The highly-substituted hybrid perovskite $\left(\mathrm{CH}\left(\mathrm{NH}_{2}\right)_{2}\right)_{0.79}\left(\mathrm{CH}_{3} \mathrm{NH}_{3}\right)_{0.16} \mathrm{Cs}_{0.05} \mathrm{~Pb}\left(\mathrm{I}_{0.83} \mathrm{Br}_{0.17}\right)_{2.97}$ maintains the perovskite structure after $>250$ hours under operating conditions, and similar substituted perovskites remain stable in devices for $>1,000$ hours. 5 ,9 These studies further demonstrate that chemical substitution preserves the advantageous optoelectronic properties of hybrid perovskites, while also allowing tunability of parameters like the band gap and phase stability. ${ }^{10}$ Chemical substitution is a powerful tool to improve the phase stability of hybrid perovskites, but the fundamental chemistry and physics describing how the substitution prevents decomposition remains underexplored.

Recent studies show that substitution at either the halide or $A$-site leads to inhibition of organic cation reorientations. Comparison of two-dimensional infrared spectroscopy data of the unsubstituted perovskites $\mathrm{CH}_{3} \mathrm{NH}_{3} \mathrm{~Pb} X_{3}(X=\mathrm{I}, \mathrm{Br}, \mathrm{Cl})$ with mixed halide perovskites demonstrated that the organic cations become partially immobilized upon halide substitution. ${ }^{11}$ Furthermore, our previous characterization of the series $\left(\mathrm{CH}_{3} \mathrm{NH}_{3}\right)_{1-x} \mathrm{Cs}_{x} \mathrm{PbBr}_{3}$ elucidated similar trends in $A$-site substituted methylammonium perovskites. ${ }^{12}$ In both cases, the breaking of local symmetry was implicated in reducing the organic dynamic degrees of freedom relative to the unsubstituted materials. $A$-site substituted materials also have higher activation energies for halide migration, 
which suggests a possible relationship between decomposition from ion migration and the organic cation dynamics. ${ }^{13}$

Organic cation dynamics are known to be linked to device performance and behavior, as they affect the optoelectronic properties of hybrid perovskites. ${ }^{14}$ Computational studies suggest that the orientation of the organic cation influences the character of the band edges through organicinorganic coupling. ${ }^{15,16}$ The liquid-like reorientations of these cations also affect the electronic excited state lifetimes of hot carriers; charge carriers excited far above the band gap relax more slowly in the hybrid perovskites $\mathrm{CH}\left(\mathrm{NH}_{2}\right)_{2} \mathrm{PbBr}_{3}$ and $\mathrm{CH}_{3} \mathrm{NH}_{3} \mathrm{PbBr}_{3}$ than in their inorganic counterpart $\mathrm{CsPBBr}_{3}$, and cation reorientations are thought to stabilize nascent polarons. ${ }^{17,18}$ The presence of a dynamic organic cation changes the relationship between the carrier density and the hot carrier cooling rates, which was attributed to changes in the phonon density between hybrid and fully organic perovskites. ${ }^{19}$ The reorientations of the organic cation are also likely to participate in dynamic, local symmetry breaking that increases the electronic excited state lifetimes through Rashba splitting of the frontier energy states. ${ }^{20,21}$ Rashba splitting may be enhanced in substituted perovskites. ${ }^{22}$

These links between phase stability, cation dynamics, and optoelectronic properties align with studies showing that the reorientations of the organic cations inform the structure and phase behavior of hybrid perovskites. In methylammonium perovskites, reductions in the dynamic degrees of freedom of methylammonium occur concurrently with decreases in symmetry within the octahedral framework. ${ }^{23,24}$ In formamidinium perovskites, the organic-inorganic coupling manifests through more incremental, complex pathways. ${ }^{25,26}$ The reorientation of formamidinium in formamidinium lead iodide $\left(\mathrm{CH}\left(\mathrm{NH}_{2}\right)_{2} \mathrm{PbI}_{3}\right)$ participates in the path-dependent phase transitions, for which the cooling rate determines whether the structure remains in the cubic perovskite phase down to $T=8.2 \mathrm{~K}$, decomposes to a non-perovskite phase, or undergoes a reentrant phase transition. ${ }^{27,28}$ The bromide analogue also undergoes complex structural changes, manifesting through five calorimetrically-observed phase transitions. ${ }^{29,30}$

We have previously described the contributions of the organic sub-lattice to the phase transitions of $\mathrm{CH}\left(\mathrm{NH}_{2}\right)_{2} \mathrm{PbBr}_{3}{ }^{30} \mathrm{CH}\left(\mathrm{NH}_{2}\right)_{2} \mathrm{PbBr}_{3}$ undergoes two crystallographic phase transitions upon cooling from room temperature. At $T=265 \mathrm{~K}$, the structure transitions from the high-temperature 
cubic phase to a tetragonal phase characterized by in-phase octahedral rotations perpendicular to the $c$ axis. Upon cooling to $T=153 \mathrm{~K}$, the tetragonal phase transitions to a low-temperature orthorhombic phase characterized by additional octahedral titling along the $c$ axis (Figure 1). ${ }^{30,31}$ The dynamic degrees of freedom of $\mathrm{CH}(\mathrm{NH})_{2}{ }^{+}$change at both crystallographic phase transitions. ${ }^{30}$ Three additional phase transitions have been observed through dilatomatry ${ }^{29}$ and heat capacity measurements, ${ }^{30}$ which neutron scattering data show are also related to the dynamic degrees of freedom of $\mathrm{CH}(\mathrm{NH})_{2}{ }^{+}$without an observable change in high resolution synchrotron X-ray or neutron crystallography at these transitions. ${ }^{30}$ These studies demonstrate that the dynamics involved in these transitions are complex and likely involve coupled motions along several axes. Furthermore, all five transitions influence the electronic transport under steady-state light illumination. ${ }^{30}$ Therefore, $\mathrm{CH}\left(\mathrm{NH}_{2}\right)_{2} \mathrm{PbBr}_{3}$ provides an excellent case study to probe how chemical substitution modifies the organic sub-lattice and how organic cation dynamics inform the overall structure.

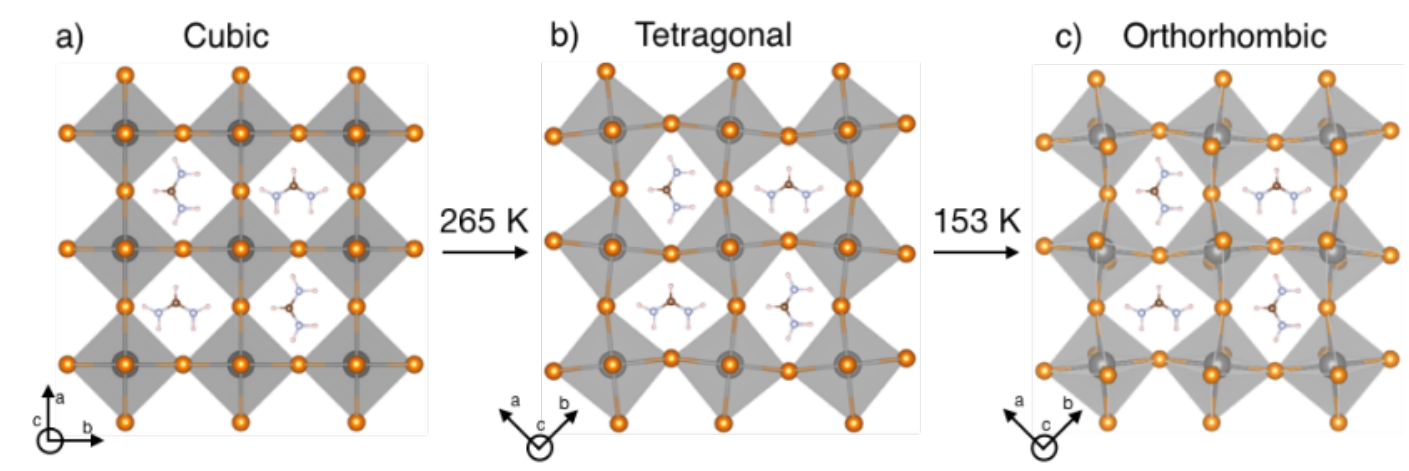

Figure 1: Octahedral tilt patterns of formamidinium lead bromide $\left(\mathrm{CH}\left(\mathrm{NH}_{2}\right)_{2} \mathrm{PbBr}_{3}\right)$ in (a) the hightemperature cubic phase (Glazer tilt: $\mathrm{a}^{0} \mathrm{a}^{0} \mathrm{a}^{0}$ ), (b) the tetragonal phase (Glazer tilt: $\mathrm{a}^{0} \mathrm{a}^{0} \mathrm{c}^{+}$), and (c) the lowtemperature orthorhombic phase (Glazer tilt: $\mathrm{a}^{-} \mathrm{b}^{-} \mathrm{c}^{-}$). Lead resides at the center of each octahedron, coordinated by six bromide ions. Formamidinium cations, which are shown in idealized positions, reside in the $A$-site void between octahedra. Transition temperatures are written above the arrows.

Nuclear magnetic resonance (NMR), nuclear quadrupole resonance (NQR), and neutron scattering spectroscopies are ideal probes to investigate the relationships between organic cation dynamics, phase transitions, and chemical substitution, as they are element-sensitive probes of local structure and dynamics. ${ }^{32-35}$ Specifically in NMR, the spin-lattice relaxation constant $\left(T_{1}\right)$, which measures the return of nuclear magnetization to equilibrium following a perturbation, is sensitive to motion on a similar timescale to the nuclear Larmor frequency and depends on both the correlation time of the motion and its nature..$^{25,36-38}$ Nuclear resonance studies also enable elucidation of the nuclear 
quadrupolar coupling tensor, characterized by a magnitude $C_{Q}$ and an asymmetry $\eta_{Q}$, which measures the coupling between the nuclear quadrupole moment and any electric field gradient present at the nucleus. Therefore, the nuclear quadrupolar coupling tensor is extremely sensitive to the symmetry of the nuclear environment (or the motionally averaged environment). The nuclear quadrupolar coupling tensor can be determined from the NMR spectrum in the case of small $C_{Q}$ constants (e.g., ${ }^{2} \mathrm{H}$ and ${ }^{14} \mathrm{~N}$ ) or from the NQR spectrum for large $C_{Q}$ constants (e.g., ${ }^{79 / 81} \mathrm{Br}$ and $\left.{ }^{127} \mathrm{I}\right),{ }^{36-41}$ although even nuclei with large $C_{\mathrm{Q}}$ constants are also becoming accessible by NMR in some cases, with sufficiently high-field magnets. ${ }^{42,43}$ Neutron scattering is a complementary technique to characterize dynamics of the organic sub-lattice, as neutrons interact strongly with hydrogen. Furthermore, the tunable energies of incident neutrons allow access to low-energy modes that are difficult to probe with optical spectroscopies. Neutron scattering has previously been used to characterize the reorientation rate and nature of the organic cation dynamics in hybrid perovskites, as well as organic-inorganic coupling. ${ }^{12,44-46}$

In the present work, we have incrementally substituted cesium for formamidinium in formamidinium lead bromide to prepare the series $\left(\mathrm{CH}\left(\mathrm{NH}_{2}\right)_{2}\right)_{1-x} \mathrm{Cs}_{x} \mathrm{PbBr}_{3}$ to elucidate the influence of chemical substitution on the temperature-dependent structure and dynamics. We confirm that in unsubstituted $\mathrm{CH}\left(\mathrm{NH}_{2}\right)_{2} \mathrm{PbBr}_{3}$, the previously observed crystallographically unresolvable phase transitions are associated with changes in the cation dynamics ${ }^{30,29}$ and nuclear resonance spectroscopies show that these dynamics correlate with local distortions in the octahedral framework at low temperatures. Cesium substitution suppresses these phase transitions, while preserving the short-range organic-organic interactions. We propose that chemical substitution by cesium disrupts the long-range order of the organic-organic interactions by introducing compressive chemical pressure, thus removing the driving force for the lowtemperature phase transitions.

\section{Results}

\section{Cation dynamics of $\mathrm{CH}\left(\mathrm{NH}_{2}\right)_{2} \mathrm{PbBr}_{3}$}

In $\mathrm{CH}\left(\mathrm{NH}_{2}\right)_{2} \mathrm{PbBr}_{3}$, neutron scattering and nuclear magnetic resonance spectroscopy (NMR) demonstrate that overall $\mathrm{CH}\left(\mathrm{NH}_{2}\right)_{2}{ }^{+}$dynamics become inhibited and slow upon cooling (Figure 
2). Mean squared displacement (MSD) values from fixed window elastic neutron scattering data decrease as a function of temperature (Figure 2a), indicating inhibited motions of the hydrogen
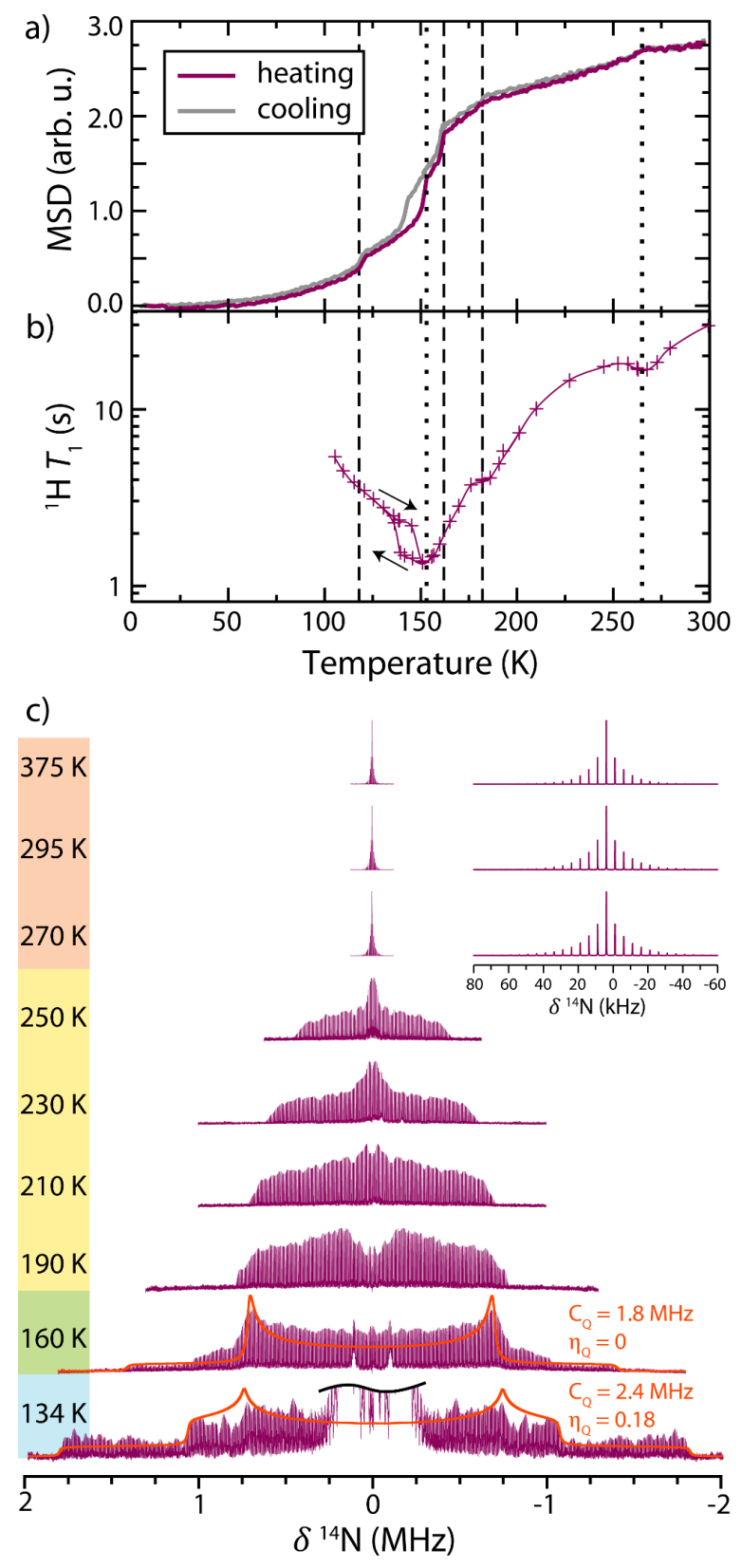

Figure 2: (a) Mean squared displacement (MSD) from fixed window elastic neutron scattering spectra for $\mathrm{CH}\left(\mathrm{NH}_{2}\right)_{2} \mathrm{PbBr}_{3}$. (b) ${ }^{1} \mathrm{H} \mathrm{NMR} T_{1}$ values as a function of temperature for $\mathrm{CH}\left(\mathrm{NH}_{2}\right)_{2} \mathrm{PbBr}_{3}$, recorded at $12.5 \mathrm{kHz}$ MAS and $14.1 \mathrm{~T}(T<200 \mathrm{~K})$ or $16.4 \mathrm{~T}(T>200 \mathrm{~K})$; the largest measured spread of sample temperatures for these measurements was $6 \mathrm{~K}$ at $200 \mathrm{~K}$. In (a) and (b) dotted lines indicate known crystallographic transitions and dashed lines indicate other phase transitions. Measurement errors are smaller than the symbols. (c) ${ }^{14} \mathrm{~N} \mathrm{NMR} \mathrm{spectra} \mathrm{of} \mathrm{CH}\left(\mathrm{NH}_{2}\right)_{2} \mathrm{PbBr}_{3}$, collected as a function of temperature. Colors on the temperature scale indicate phase transitions. ${ }^{29,30}$ Spectra at $T=270 \mathrm{~K}$ and above were recorded at $5 \mathrm{kHz}$ MAS using a Hahn echo pulse sequence, while those at and below $250 \mathrm{~K}$ were recorded under static conditions using a WURST CPMG pulse sequence with VOCS acquisition. The truncated signals in the $T=134 \mathrm{~K}$ spectrum are due to probe ringing; this was removed from the other WURST 
CPMG spectra by not recording the first two echoes. However, the short spin-spin relaxation $\left(T_{2}\right)$ constant made similar modifications impossible for the $134 \mathrm{~K}$ spectrum due to insufficient signal-tonoise if the first two echoes were removed.

atoms, and thus the organic cations, as the sample is cooled. Figure $2 \mathrm{~b}$ shows the ${ }^{1} \mathrm{H}$ spin-lattice relaxation constant $\left(T_{1}\right)$ as a function of temperature. Assuming idealized reorientations according to Bloembergen-Purcell-Pound (BPP) theory, ${ }^{47}$ the $T_{1}$ relates to the correlation time of motion through the spectral density as:

$$
\begin{aligned}
& \frac{1}{T_{1}}=A\left(J\left(\omega_{0}\right)+4 J\left(2 \omega_{0}\right)\right) \\
& J(\omega)=\frac{\tau_{c}}{1+\omega^{2} \tau_{c}^{2}}
\end{aligned}
$$

where $J(\omega)$ is the spectral density, $\omega_{0}$ is the Larmor frequency, $\tau_{c}$ is the correlation time of the motion, and the pre-factor $A$ is given by the magnitude of the oscillations in the local field and depends on the nature of the motion. The Lorentzian form of the spectral density predicted by BPP theory explains the overall trends in the data: from room temperature, the $T_{1}$ decreases with decreasing temperature since the cation rotation is in the fast-motion regime $\left(\omega_{0} \tau_{c} \ll 1\right.$ and $T_{1} \propto$ $\left.1 / \tau_{c},\right)$. This trend continues until at $\sim 150 \mathrm{~K}$ the $T_{1}$ exhibits a minimum as the motion rate approaches the Larmor frequency $\left(\tau_{c} \sim 1 \mathrm{~ns}\right)$. Below $\sim 150 \mathrm{~K}$ the $T_{1}$ then increases with decreasing temperature since the rotation is in the slow-motion regime $\left(\omega_{0} \tau_{c} \gg 1\right.$ and $\left.T_{1} \propto \tau_{c}\right)$.

Overlaid on these general trends, anomalies in the MSD and ${ }^{1} \mathrm{H} T_{1}$ data at $T=265 \mathrm{~K}, 182 \mathrm{~K}, 162$ $\mathrm{K}, 153 \mathrm{~K}$ and $T=118 \mathrm{~K}$ correlate with previously reported thermodynamic phase transitions. ${ }^{29-31}$ At $T=265 \mathrm{~K}, \mathrm{CH}\left(\mathrm{NH}_{2}\right)_{2} \mathrm{PbBr}_{3}$ transitions from the cubic perovskite phase to a tetragonal perovskite phase, ${ }^{29,31}$ which appears as an anomaly in the MSD data in Figure $2 b$, and as a dip in the ${ }^{1} \mathrm{H} T_{1}$. This dip is ascribed to a convolution of the discontinuity caused by a first order transition ${ }^{48}$ with magic angle spinning (MAS) induced temperature gradients. The MSD and ${ }^{1} \mathrm{H} T_{1}$ exhibit similar features at the $T=153 \mathrm{~K}$ tetragonal to orthorhombic phase transition. This transition also exhibits hysteresis that is indicative of a sluggish first order phase transition; the extent of the hysteresis is slightly different in the MSD and NMR measurements, most likely due to different cooling rates. While they do not manifest crystallographically, the three additional phase transitions display anomalies in the MSD, indicating that they relate to the dynamic degrees of freedom of $\mathrm{CH}\left(\mathrm{NH}_{2}\right)_{2}{ }^{+}$. The kink in the ${ }^{1} \mathrm{H} T_{1}$ at the $T=182 \mathrm{~K}$ phase transition suggests second order character. ${ }^{48}$ Any features associated with the $T=162 \mathrm{~K}$ and $118 \mathrm{~K}$ phase transitions in the ${ }^{1} \mathrm{H} T_{1}$ are too subtle to resolve. The ${ }^{1} \mathrm{H} T_{1}$ and neutron MSD data demonstrate that the phase 
transitions of $\mathrm{CH}\left(\mathrm{NH}_{2}\right)_{2} \mathrm{PbBr}_{3}$ correlate to modulation of the dynamic degrees of freedom of $\mathrm{CH}\left(\mathrm{NH}_{2}\right)_{2}{ }^{+}$. These data are consistent with the previously observed changes in shape of the electronic density at the $A$-site in Fourier difference maps calculated from high resolution synchrotron X-ray diffraction. ${ }^{30}$

Further details about the changes to $\mathrm{CH}\left(\mathrm{NH}_{2}\right)_{2}{ }^{+}$dynamic degrees of freedom are evident in the nuclear quadrupolar coupling observed in the ${ }^{14} \mathrm{~N}$ NMR spectra. In the absence of any rotation, the calculated $C_{Q}$ of a formamidinium ${ }^{14} \mathrm{~N}$ is $2.8 \mathrm{MHz}$ (further details in the Supporting Information). In the high-temperature cubic phase, the ${ }^{14} \mathrm{~N}$ NMR spectra exhibit nearly isotropic tumbling, with a spectral width corresponding to effective $C_{Q}$ constants less than $50 \mathrm{kHz}$. The lack of a single $I=$ 1 nuclear quadrupolar line-shape indicates a distribution of cation motions with different anisotropies. This line-shape is conserved at higher temperatures, signifying that the nature of the motion remains unchanged, despite the changes in reorientation rate apparent in the ${ }^{1} \mathrm{H} T_{1}$. Upon cooling, the cubic to tetragonal phase transition at $T=265 \mathrm{~K}$ results in a greater anisotropy of tumbling and $C_{Q}$ constants are observed of up to $\sim 600 \mathrm{kHz}$, with increasing anisotropy as the temperature decreases. The lack of a single nuclear quadrupolar line-shape still indicates a distribution of motions. In contrast, below the crystallographically-unresolvable $T=182 \mathrm{~K}$ phase transition, a subset of signals can be distinguished with a well-defined nuclear quadrupolar lineshape $\left(C_{Q}=1.8 \mathrm{MHz}, \eta_{Q}=0\right)$, in addition to a signal with a distribution of $C_{Q}$ constants, akin to the higher temperature spectra. Therefore, by this temperature, a component of the reorientational dynamics has been frozen out for a population of $\mathrm{CH}\left(\mathrm{NH}_{2}\right)_{2}{ }^{+}$. The ${ }^{14} \mathrm{~N}$ NMR spectrum collected at $T=134 \mathrm{~K}$, below the tetragonal to orthorhombic phase transition and above the $T=118 \mathrm{~K}$ crystallographically unresolvable phase transition is characterized by only one nuclear quadrupolar tensor, indicating that all the cations are now undergoing the same type of motion. The increase in the $C_{Q}$ to $2.4 \mathrm{MHz}$ with $\eta_{Q}=0.18$, close to the calculated values for static formamidinium, indicates that overall reorientations have been frozen out, leaving only libration. Constricted or slow molecular motions are consistent with the quasielastic neutron scattering spectra; between $T$ $=130 \mathrm{~K}$ and $110 \mathrm{~K}$ any organic cation reorientations or librations become slower than the resolution of the instrument $( \pm 1 \mu \mathrm{eV}$, or a residence time $\gtrsim 4 \mathrm{~ns}$; Figure S6), consistent with observations of the ${ }^{1} \mathrm{H} T_{1}$ (Figure $2 \mathrm{~b}$ ). 
Bromine nuclear quadrupolar resonance (NQR) spectra (Figure 3a) suggest that the organic dynamic degrees of freedom couple to local distortions of the octahedral framework during the crystallographically-unresolvable phase transitions of $\mathrm{CH}\left(\mathrm{NH}_{2}\right)_{2} \mathrm{PbBr}_{3}$ that manifest primarily in changes of the cation dynamics. Previous total scattering measurements of methylammonium lead bromide have suggested that the orthorhombic distortion is still present in the higher symmetry phases, but is dynamically or configurationally

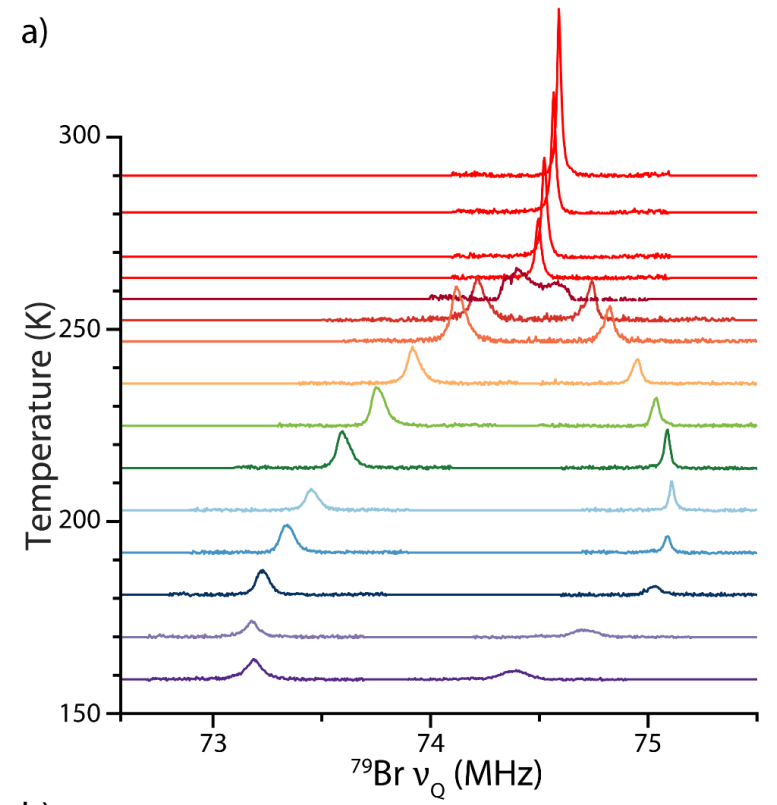

b)

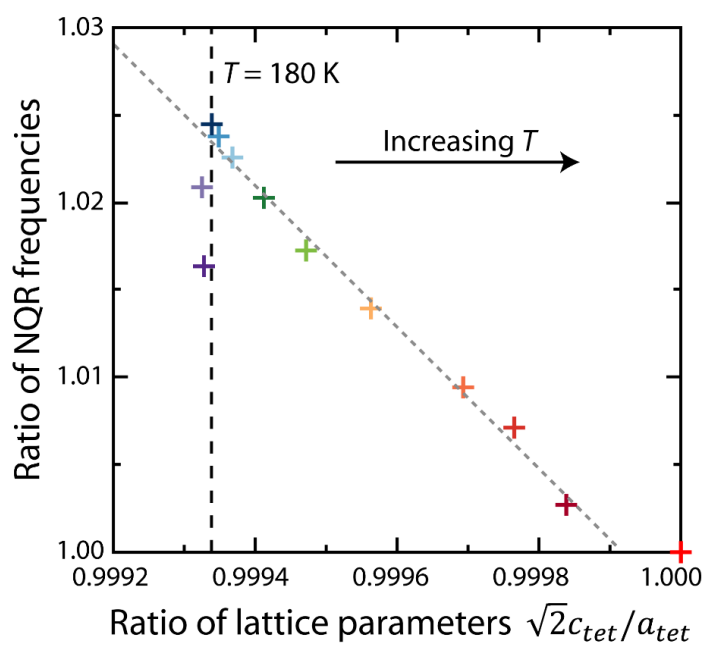

Figure 3: (a) ${ }^{79} \mathrm{Br}$ NQR spectra collected on $\mathrm{CH}\left(\mathrm{NH}_{2}\right)_{2} \mathrm{PbBr}_{3}$. The $T=265 \mathrm{~K}$ phase transition results in peak splitting. The relative intensities of each resonance indicate that the higher frequency (lower intensity) signal relates to the bromine site on the unique $c$ axis. (b) Ratio of the frequency of the NQR signals compared to the degree of tetragonal compression given by the ratio of the pseudocubic lattice parameters, 
$\sqrt{2} c_{t e t} / a_{t e t}$ according to the relationships $\sqrt{2} a_{c u b}=a_{t e t}$ and $a_{c u b}=c_{t e t}$. The dashed grey line represents a linear relationship. The vertical dashed line indicates the phase transition temperature. Measurement errors are smaller than the symbols.

averaged. ${ }^{49}$ However, the single resonance observed at temperatures above the cubic to tetragonal phase transition indicates that all bromine environments are equivalent; therefore, any dynamic distortion is faster than the resultant separation of the NQR signals (at least $100 \mathrm{kHz}$ ). The general trend of decreasing quadrupolar frequency $\left(v_{Q}-\right.$ given by $C_{Q} / 2$ in axial environments) on cooling occurs because the anisotropy of the bromine environment is reduced by lattice contraction. At the cubic to tetragonal phase transition at $T=265 \mathrm{~K}$, the NQR signal splits into two distinct bromine environments, consistent with the reduction in crystallographic symmetry. Above the $T=182 \mathrm{~K}$ crystallographically-unresolvable phase transition, the ratio of $v_{Q}$ for the two signals depends linearly on the magnitude of the crystallographic tetragonal compression, quantified as the ratio of the lattice parameters $\sqrt{2} c_{\text {tet }} / a_{\text {tet }}$ (Figure $3 b$ ). We note that the tetragonal compression of the unit cell $(c / a<1)$ necessitates a large compression of the apical lead-bromide bonds relative to the shrinking of $a_{t e t} / \sqrt{ } 2$ due to the octahedral tilting, akin to the $P 4 / \mathrm{mbm}$ phase of $\mathrm{AgTaO}_{3}$ and the pseudo-tetragonal Imma phase of $\mathrm{SrZrO}_{3} .{ }^{50,51}$ Below $182 \mathrm{~K}$ the higher frequency peak assigned to the $c$ axis bromide in the NQR spectra begins to decrease in frequency. As the ratio of the nuclear quadrupolar frequencies deviates from a linear relationship with the $\sqrt{2} c_{t e t} / a_{t e t}$ ratio, contraction of the inorganic framework is insufficient to explain the data. Below the crystallographically unresolvable phase transition at $T=162 \mathrm{~K}$ no NQR signal could be determined between 60 and 80 $\mathrm{MHz}$, which indicates a significant change in the bromine environment. The disappearance of the NQR signal could be due to rapid relaxation induced by the cation motion, the timescale of which is approaching the NQR frequency as the temperature decreases (vide infra), or to a wide distribution of environments leading to signals that lie below the signal-to-noise ratio; if not motionally averaged, the orientation of adjacent $\mathrm{CH}\left(\mathrm{NH}_{2}\right)_{2}{ }^{+}$cations can induce large changes to the local bromine environment (Figure S1). As the MSD and ${ }^{1} \mathrm{H} T_{1}$ data demonstrate a change in the organic cation dynamics at $182 \mathrm{~K}$ and $162 \mathrm{~K}$, the changes in the bromine environments exhibited in the NQR spectra likely arise from bromine- $\mathrm{CH}\left(\mathrm{NH}_{2}\right)_{2}{ }^{+}$interactions. 


\section{Effect of Cesium Substitution}

The phase behavior of the series $\left(\mathrm{CH}\left(\mathrm{NH}_{2}\right)_{2}\right)_{1-x} \mathrm{Cs}_{x} \mathrm{PbBr}_{3}$ also suggests that the $A$-site cation actively participates in the phase transitions of $\mathrm{CH}\left(\mathrm{NH}_{2}\right)_{2} \mathrm{PbBr}_{3}$, as partial cesium substitution suppresses the low-temperature phase transitions. All members of the series with $x<0.4$ crystallize in the high-temperature cubic phase. Any substituted sample with $x>0.4$ is phase separated between a substituted cubic perovskite phase and an orthorhombic $\mathrm{CsPbBr}_{3}$ phase. Phase separated materials were not investigated further. On cooling, all single-phase materials undergo the cubic to tetragonal phase transition, which is observed in the high-resolution synchrotron X-ray diffraction (SXRD) data (Figure S3, S4). In contrast, SXRD data do not show any change in the characteristic region between $Q=1.5 \AA^{-1}$ and $2.1 \AA^{-1}$ to indicate a tetragonal to orthorhombic phase transition between room temperature and $T=100 \mathrm{~K}$ (Figure S3) for the substituted samples. However, the characteristic features of the orthorhombic phase in $\mathrm{CH}\left(\mathrm{NH}_{2}\right)_{2} \mathrm{PbBr}_{3}$ have low, pathdependent intensities, so it may simply not be possible to resolve them. ${ }^{30}$ 

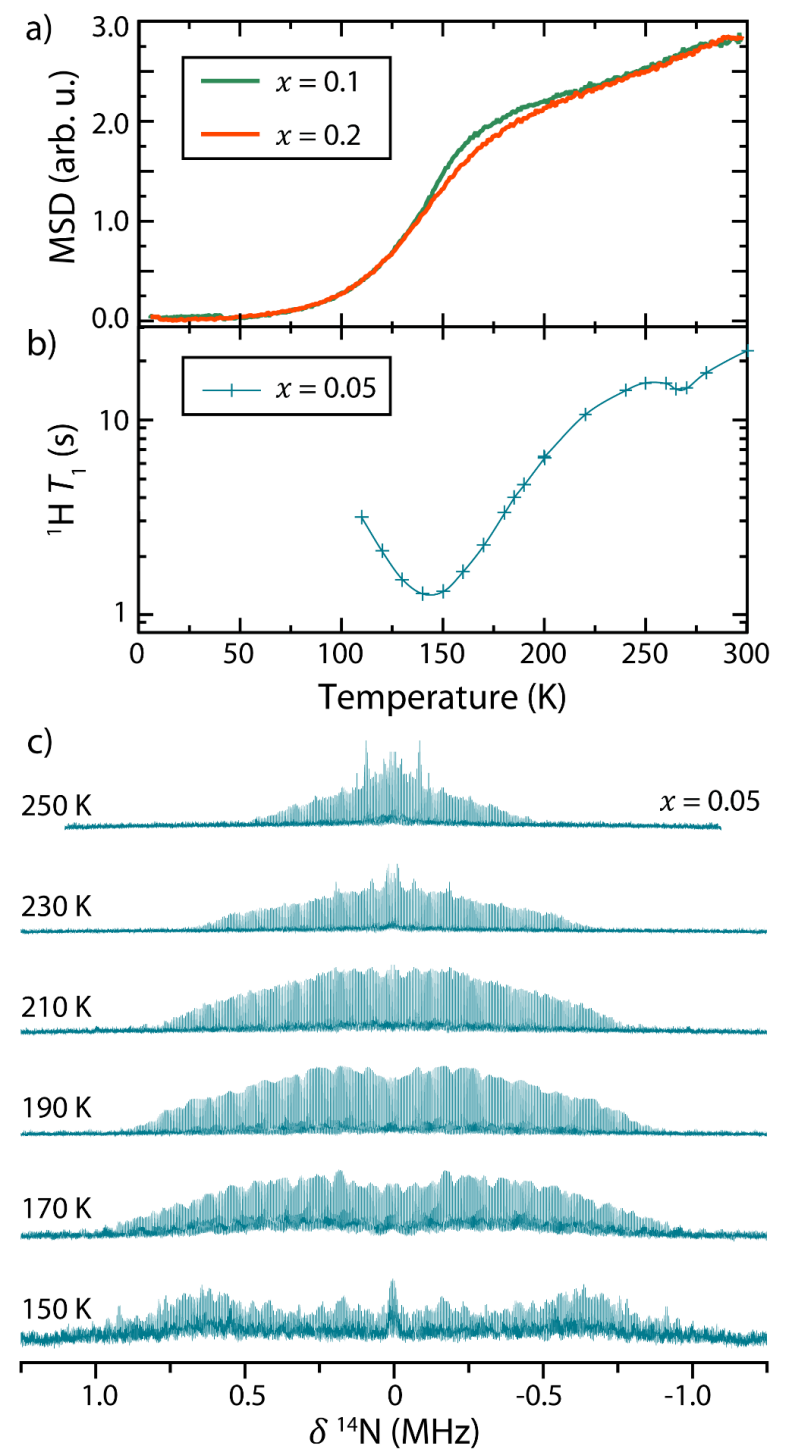

Figure 4: (a) Mean squared displacement (MSD) extracted from fixed window elastic neutron scattering spectra for $\left(\mathrm{CH}\left(\mathrm{NH}_{2}\right)_{2}\right)_{1-x} \mathrm{Cs}_{x} \mathrm{PbBr}_{3}$, where $x=0.1$ and 0.2 . (b) ${ }^{1} \mathrm{H}$ NMR $T_{l}$ values as a function of temperature for $\left(\mathrm{CH}\left(\mathrm{NH}_{2}\right)_{2}\right)_{0.95} \mathrm{Cs}_{0.05} \mathrm{PbBr}_{3}$. (c) ${ }^{14} \mathrm{~N}$ NMR spectra of $\left(\mathrm{CH}\left(\mathrm{NH}_{2}\right)_{2}\right)_{0.95} \mathrm{Cs}_{0.05} \mathrm{PbBr}_{3}$ as a function of temperature, recorded under static conditions with VOCS WURST CPMG acquisition.

Characterization of the organic cation dynamics reveals an absence of any signatures of the four low-temperature phase transitions in $\left(\mathrm{CH}\left(\mathrm{NH}_{2}\right)_{2}\right)_{1-x} \mathrm{Cs}_{x} \mathrm{PbBr}_{3}$ with $x>0$. The cubic to tetragonal phase transition manifests with features in the MSD, ${ }^{1} \mathrm{H} T_{1}$ values, and ${ }^{14} \mathrm{~N}$ NMR spectra; aside from these features, however, there is no indication of further phase transitions (Figure 4). For $\left(\mathrm{CH}\left(\mathrm{NH}_{2}\right)_{2}\right)_{1-x} \mathrm{Cs}_{x} \mathrm{PbBr}_{3}$ with $x=0.1$ and 0.2 , the MSD decreases smoothly with temperature below the cubic to tetragonal phase transition. Similarly, with as little as $5 \% \mathrm{Cs}^{+}$substitution, the features in the ${ }^{1} \mathrm{H} T_{1}$ that correlate to the low-temperature $(T \leq 182 \mathrm{~K})$ phase transitions do not appear. ${ }^{14} \mathrm{~N}$ NMR spectra for $\left(\mathrm{CH}\left(\mathrm{NH}_{2}\right)_{2}\right)_{0.95} \mathrm{Cs}_{0.05} \mathrm{PbBr}_{3}$ preserve the general trend of increasing 
anisotropy $\left(C_{Q}\right)$ with decreasing temperature in $\mathrm{CH}\left(\mathrm{NH}_{2}\right)_{2} \mathrm{PbBr}_{3}$, but the component with a distinct nuclear quadrupolar line-shape observed for $\mathrm{CH}\left(\mathrm{NH}_{2}\right)_{2} \mathrm{PbBr}_{3}$ below $T=182 \mathrm{~K}$ is not observed in the $170 \mathrm{~K}$ spectrum. There is some evidence of a more defined environment near $T=150 \mathrm{~K}$ with $C_{Q} \approx 1.8 \mathrm{MHz}$, but the features are approximately $100 \mathrm{kHz}$ broader than for the unsubstituted perovskite. Together, these data demonstrate that $\mathrm{Cs}^{+}$substitution suppresses the low-temperature phase transitions of $\mathrm{CH}\left(\mathrm{NH}_{2}\right)_{2} \mathrm{PbBr}_{3}$. A summary phase diagram is presented in Figure 5.

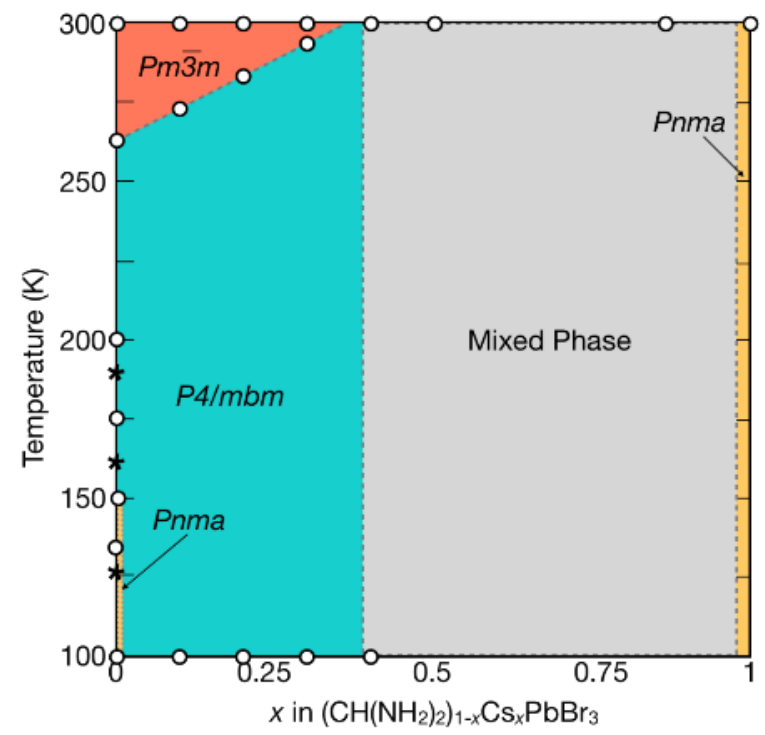

Figure 5: The phase behavior of the series $\left(\mathrm{CH}\left(\mathrm{NH}_{2}\right)_{2}\right)_{1-x} \mathrm{Cs}_{x} \mathrm{PbBr}_{3}$, constructed from crystallography, neutron spectroscopy, and solid-state NMR spectroscopy. Black asterisks indicate crystallographically unresolvable phase transitions and open white circles indicate data collection temperatures near phase transition temperatures. The increase in cubic-tetragonal phase transition temperature as a function of $x$ results from the substitution of a smaller cation in the $A$-site void.

Cesium substitution increases the number of distinct local environments in $\left(\mathrm{CH}\left(\mathrm{NH}_{2}\right)_{2}\right)_{1-x} \mathrm{Cs}_{x} \mathrm{PbBr}_{3}$, evidenced by ${ }^{14} \mathrm{~N}$ NMR spectra (Figure 6a) and ${ }^{79} \mathrm{Br} \mathrm{NQR}$ spectra (Figure 6b) collected for all samples in the high-temperature cubic phase. The ${ }^{14} \mathrm{~N}$ NMR spectra broaden with increasing substitution due to components with larger $C_{Q}$ values. The broad features indicate increasing anisotropy of cation rotations with $x$, likely due to the introduction of local distortions from $\mathrm{Cs}^{+}$substitution. This anisotropy extends to the inorganic framework. With just $5 \% \mathrm{Cs}^{+}$ substitution, the sharp peak observed in the ${ }^{79} \mathrm{Br} \mathrm{NQR}$ data of $\mathrm{CH}\left(\mathrm{NH}_{2}\right)_{2} \mathrm{PbBr}_{3}$ broadens and splits into multiple signals, corresponding to different local environments throughout the bulk of the sample. Specifically, the broad signals at $\sim 74.4,73.6$ and $72.8 \mathrm{MHz}$ are ascribed to ${ }^{79} \mathrm{Br}$ environments with zero, one and two $\mathrm{Cs}^{+}$nearest neighbors, respectively. Increasing the level of substitution leads to a corresponding increase of the lower-frequency signals, as the probability of 
having $\mathrm{Cs}^{+}$in the immediate coordination shells increases. By $x=0.35$ only a broad featureless resonance is observed, corresponding to an effectively continuous distribution of possible local environments due to the different possible configurations of $\mathrm{Cs}^{+}$substitution in the immediate and extended coordination shells. This NQR broadening is consistent with the microstrain detected from XRD peak broadening that is present in all substituted materials (Figure S5); the NQR broadening indicates that the local $\mathrm{Br}$ environment experiences additional changes to the local electric field gradient beyond microstrain.
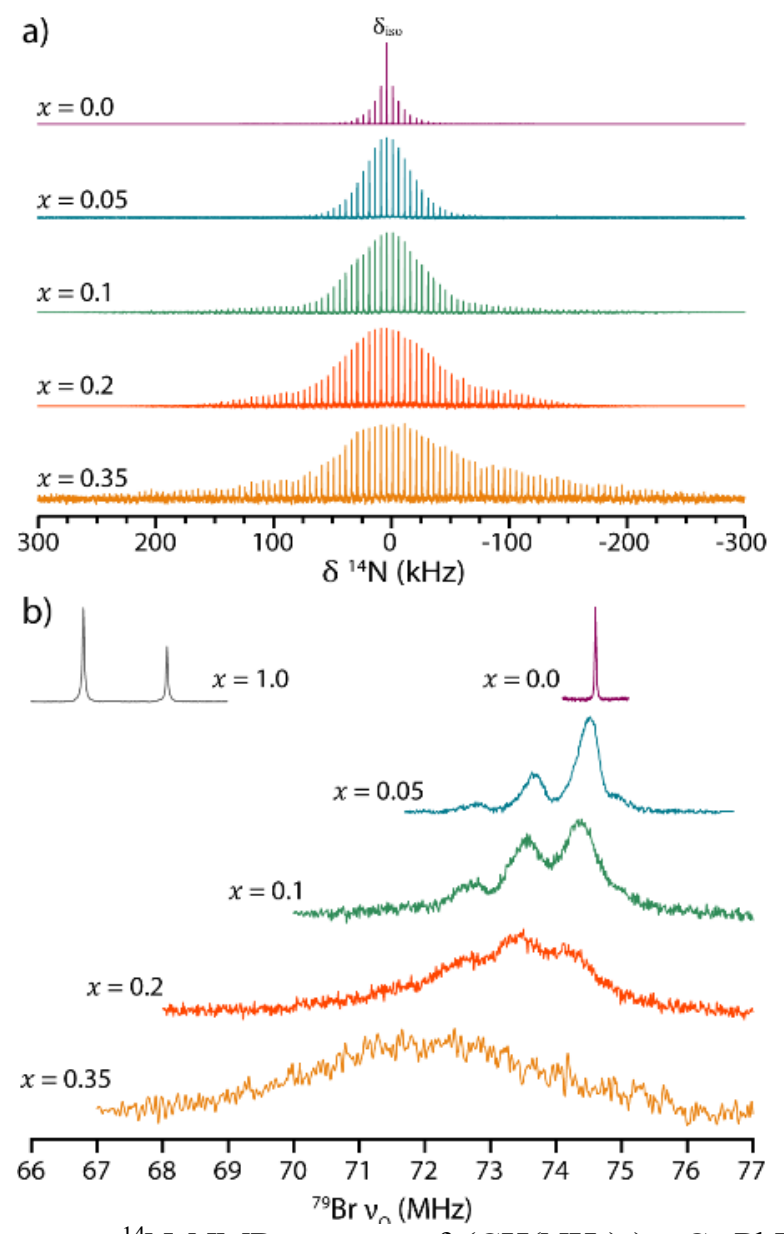

Figure 6: (a) Room-temperature ${ }^{14} \mathrm{~N}$ NMR spectra of $\left(\mathrm{CH}\left(\mathrm{NH}_{2}\right)_{2}\right)_{1-x} \mathrm{Cs}_{x} \mathrm{PbBr}_{3}$, where $x=0$ to 0.35 , recorded at $16.4 \mathrm{~T}$ and $5 \mathrm{kHz}$ MAS using a Hahn echo pulse sequence. (b) Room-temperature ${ }^{79} \mathrm{Br}$ NQR spectra collected on $\left(\mathrm{CH}\left(\mathrm{NH}_{2}\right)_{2}\right)_{1-x} \mathrm{Cs}_{x} \mathrm{PbBr}_{3}$ with VOCS acquisition in steps of $200 \mathrm{kHz}$. Data for $\mathrm{CsPbBr}_{3}$ are presented in the top left corner. 


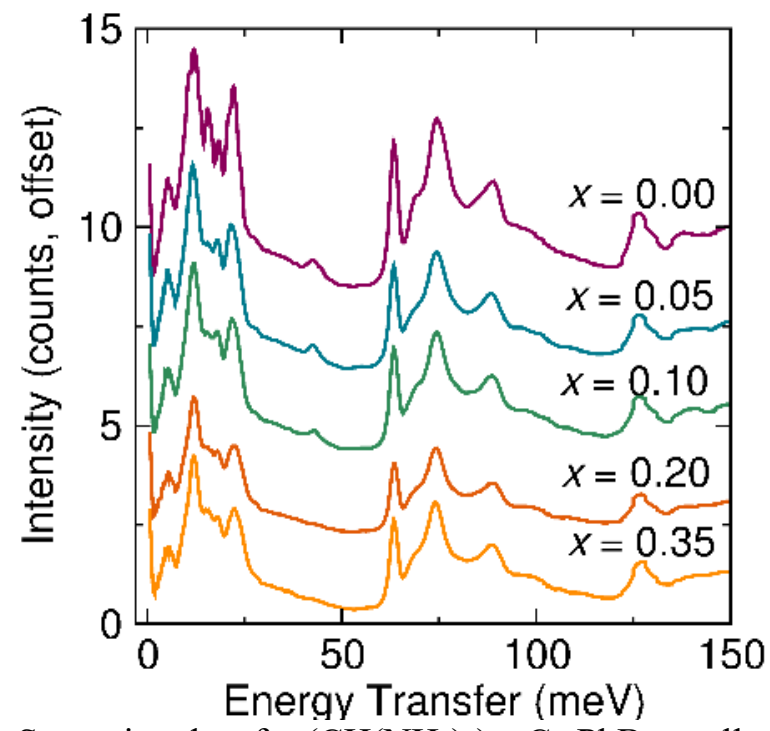

Figure 7: Inelastic Neutron Scattering data for $\left(\mathrm{CH}\left(\mathrm{NH}_{2}\right)_{2}\right)_{1-x} \mathrm{Cs}_{x} \mathrm{PbBr}_{3}$, collected at $T=5 \mathrm{~K}$ showing that chemical substitution has a minimal influence on the internal vibrational modes of formamidinium. Phonon calculations indicate that the low-energy modes $(<30 \mathrm{meV})$ correspond to coupled organic cation librations and octahedral tilting, and high-energy modes $(>50 \mathrm{meV})$ correspond to internal organic cation vibrations.

Although $\mathrm{Cs}^{+}$substitution increases the disorder within the inorganic framework, the local behavior of $\mathrm{CH}\left(\mathrm{NH}_{2}\right)_{2}{ }^{+}$appears to be preserved. Spectroscopic characterization of the vibrational modes of $\left(\mathrm{CH}\left(\mathrm{NH}_{2}\right)_{2}\right)_{1-x} \mathrm{Cs}_{x} \mathrm{PbBr}_{3}$ with inelastic neutron scattering (INS) demonstrates that the vibrational landscape around $\mathrm{CH}\left(\mathrm{NH}_{2}\right)_{2}{ }^{+}$is similar across the series of $\left(\mathrm{CH}\left(\mathrm{NH}_{2}\right)_{2}\right)_{1-x} \mathrm{Cs}_{x} \mathrm{PbBr}_{3}$ compounds, as the major features in the INS spectra are conserved (Figure 7). Several features are damped upon substitution, primarily the features near $15 \mathrm{meV}$ that broaden with increasing $x$ and near $45 \mathrm{meV}$ that cannot be resolved for $x>0.1$ (Figure S8). These modes correspond lead-bromide bending coupled to formamidinium librations, similar to those in related perovskites, ${ }^{52-54}$ as confirmed by the calculated phonon spectrum (Figure S9). Relative to the series of compounds $\left(\mathrm{CH}_{3} \mathrm{NH}_{3}\right)_{1-x} \mathrm{Cs}_{x} \mathrm{PbBr}_{3}$, which form an orientational glass on substitution, ${ }^{12}$ these changes are minor and correlate to the changes in organic-inorganic coupling also revealed by the ${ }^{79} \mathrm{Br} \mathrm{NQR}$. The rates of cation reorientations also remain similar as a function of $x$ (Figure 8), where the correlation times from the ${ }^{1} \mathrm{H} T_{1}$ values have been predicted using BPP theory (details in SI). Furthermore, the apparent activation energies for cation motion extracted from Arrhenius plots of the correlation times change by less than $0.02 \mathrm{eV}$ as a function of $x$ (Figure 8). Above 140 $\mathrm{K}$, the activation energy of cation motions $\left(E_{a}\right)$ for $\left(\mathrm{CH}\left(\mathrm{NH}_{2}\right)_{2}\right)_{1-x} \mathrm{Cs}_{x} \mathrm{PbBr}_{3}$ with $x=0.00,0.05$ and 0.35 is close to $0.10 \mathrm{eV}$; below $\sim 140 \mathrm{~K} E_{a}$ decreases to $0.05 \mathrm{eV}$. The reduction in activation energy 
at lower temperature may be associated with the freezing out of overall rotation observed by ${ }^{14} \mathrm{~N}$ NMR at the $T=153 \mathrm{~K}$ transition. The preservation of this reduction in activation energy with chemical substitution implies that the same freezing still occurs in the substituted materials, but not cooperatively and perhaps over a range of temperatures rather than as a sharp transition, due to the heterogeneity in the local environment. The change in apparent activation energy occurs at approximately the minimum in the ${ }^{1} \mathrm{H} T_{1}$ (Figure $3 \mathrm{~b}$ ), and as such the $T_{1}$ minimum is asymmetric (Figure S3). An alternative explanation for the change in gradient is therefore non-BPP behavior, rather than a change in the true activation energy; ${ }^{55}$ see Supplementary Information for further discussion.

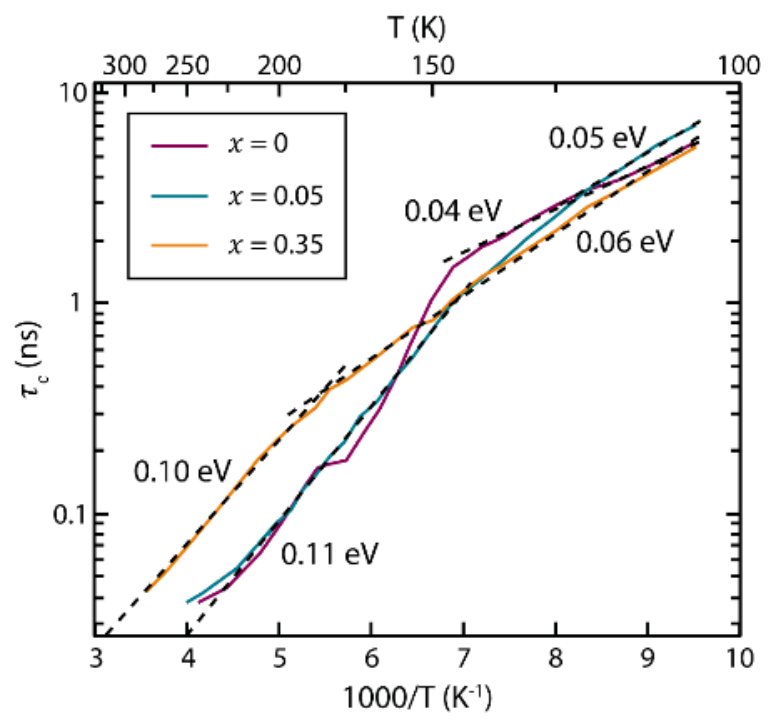

Figure 8: (a) Correlation times extracted from the ${ }^{1} \mathrm{H}$ NMR $T_{1}$ collected as a function of temperature for $\left(\mathrm{CH}\left(\mathrm{NH}_{2}\right)_{2}\right)_{1-x} \mathrm{Cs}_{x} \mathrm{PbBr}_{3}$, where $x=0.00,0.05$, and 0.35 . Details about the derivation of the correlation times are presented in the SI. The dashed lines represent linear fits.

\section{Discussion}

We propose that organic-organic interactions contribute to the four low-temperature phase transitions of $\mathrm{CH}\left(\mathrm{NH}_{2}\right)_{2} \mathrm{PbBr}_{3}$ that involve concerted changes in the cation dynamics. At each phase transition temperature the MSD, ${ }^{1} \mathrm{H} T_{1}$, and ${ }^{14} \mathrm{~N}$ NMR data demonstrate a change in the dynamic degrees of freedom of $\mathrm{CH}\left(\mathrm{NH}_{2}\right)_{2}{ }^{+}$and the anisotropy of the $A$-site void. This does not necessarily indicate positional long-range ordering of the organic sub-lattice; rather, these transitions are characterized by a cooperative change in cation dynamics. While ${ }^{79} \mathrm{Br}$ NQR data demonstrate that the octahedra also experience changes in their local environment at these 
temperatures, inter-octahedral interactions are unlikely to drive the phase transitions at $T=182 \mathrm{~K}$, $162 \mathrm{~K}$, and $118 \mathrm{~K}$, as no analogous transitions occur in related hybrid perovskites. ${ }^{12,45}$ The temperature-dependence observed in the NQR spectra are likely a response of the lead-bromide octahedra to changes in the $\mathrm{CH}\left(\mathrm{NH}_{2}\right)_{2}{ }^{+}$dynamics. The frequency ratio between the two NQR signals in the tetragonal phase correlates closely with the ratio of lattice parameters $\left(\sqrt{2} c_{t e t} / a_{t e t}\right)$ until below the $T=182 \mathrm{~K}$ phase transition, at which point the two ratios deviate dramatically (Figure 3b). Therefore, while lattice compression accounts for the changes in local bromide environment initially, below $182 \mathrm{~K}$ another interaction must lead to the observed peak shift. As this transition occurs concurrently with a reduction in the dynamic degrees of freedom of $\mathrm{CH}\left(\mathrm{NH}_{2}\right)_{2}{ }^{+}$, organic-inorganic coupling most likely leads to a change in the local bromide environment. A similar argument can be made for the signal change at $T=162 \mathrm{~K}$ and emphasizes the role of the organic cation in the low-temperature phase transitions. These changes in the bromine environment also explain why transitions are observed in the photoconductivity at the crystallographically-unresolvable phase transitions primarily associated with changes in the cation dynamics. ${ }^{30}$ Currently available scattering-based investigation of the local structure, for example with pair distribution function analysis, is unlikely to resolve these transitions owing to their dynamical and subtle nature. It would be possible to study changes in the local bromine environments using elastically-resolved diffuse scattering, as is currently available with modern neutron scattering instrumentation. Otherwise, we propose that spatially-resolved techniques may be needed to further elucidate the nature of these phase transitions.

Organic-organic interactions appear quite sensitive to disruption from $\mathrm{Cs}^{+}$substitution. As little as $5 \% \mathrm{Cs}^{+}$substitution suppresses any signature of the four low temperature phase transitions in the crystallography, MSD, ${ }^{1} \mathrm{H} T_{1}$, and ${ }^{14} \mathrm{~N}$ NMR. We cannot rule out the presence of sluggish phase transitions that have obscured signatures outside the sensitivity of our techniques; however, we do not observe features characteristic of glassy dynamics or other short-range ordered phenomena, which we and others have previously observed in related systems. ${ }^{12,57}$ Therefore, we conclude that the phase transitions are suppressed in the cesium substituted samples below the cubic to tetragonal phase transition. While these data indicate a lack of cooperative changes in the dynamic degrees of freedom in substituted samples, $\mathrm{CH}\left(\mathrm{NH}_{2}\right)_{2}{ }^{+}$molecules exhibit similar local behavior across the 
series $\left(\mathrm{CH}\left(\mathrm{NH}_{2}\right)_{2}\right)_{1-x} \mathrm{Cs}_{x} \mathrm{PbBr}_{3}$, with similar temperature dependent reorientation rates and internal vibration energies.

Describing these interactions necessitates an understanding of the driving forces behind perovskite phase transitions. Oxide and halide perovskite phase transitions generally occur to maximize coordination of the $A$-site cation by the octahedral framework. ${ }^{58}$ In hybrid perovskites, the organic cations can coordinate the octahedral units through hydrogen bonds, ${ }^{25,59}$ but unlike $\mathrm{CH}_{3} \mathrm{NH}_{3}{ }^{+}$ perovskites, the geometry and coordination of the lead-bromide octahedra conflict with the geometry and electrostatic interactions of $\mathrm{CH}\left(\mathrm{NH}_{2}\right)_{2}{ }^{+}$. The hydrogen-bonding pattern of $\mathrm{CH}\left(\mathrm{NH}_{2}\right)_{2}{ }^{+}$is not compatible with the geometry of the bromide hydrogen bonding acceptors and appears to have more complex molecule-cage interactions than for $\mathrm{CH}_{3} \mathrm{NH}_{3}{ }^{+}$counterparts. ${ }^{57}$ Furthermore, $\mathrm{CH}\left(\mathrm{NH}_{2}\right)_{2}{ }^{+}$has a weak electrostatic dipole $(\mathrm{p}=0.35 \mathrm{D})$ but a strong molecular electrostatic quadrupole $\left(\left|\mathrm{Q}_{11}\right|=18.3 \mathrm{D} \cdot \AA\right)$ due to the cancellation of the strong bond dipoles between carbon and each nitrogen (cf., $\mathrm{CH}_{3} \mathrm{NH}_{3}{ }^{+}, \mathrm{p}=2.3 \mathrm{D},\left|\mathrm{Q}_{11}\right|=3.6 \mathrm{D} \cdot \AA$ ).$^{60}$ The quadrupolar moments of formamidinium cations yield a strain field due to electrostatic interactions with the inorganic octahedra (Figure 9a,b), where positive charge attracts the anionic sublattice and negative charge repulses the anionic sublattice. These strain fields are analogous to chemical pressure, as has been visualized in intermetallic compounds, ${ }^{61}$ and are mathematically equivalent to elastic dipoles (a second rank tensor). ${ }^{62}$ Pairs of axially-symmetric electrostatic quadrupoles (and elastic dipole tensors in a homogeneous dielectric) have a preference to order in a "T" shape due to their compatible compressive and expansive strain fields (Figure 9c); this "T"-shape ordering can tile in two dimensions (Figure 9d), however such local ordering cannot geometrically tile space in three dimensions. ${ }^{30,62}$ The electrostatic quadrupole of formamidinium is not axially symmetric, but there are nevertheless many possible low-energy 3D orientational arrangements, and we previously showed that the resulting geometric frustration leads to the cation-orientation mediated phase transitions. ${ }^{30,62}$ This aligns with the recent description of $\mathrm{CH}\left(\mathrm{NH}_{2}\right)_{2} \mathrm{PbBr}_{3}$ as an incipient ferroelastic, ${ }^{63}$ as a uniform strain field causes elastic dipoles to spontaneously align. ${ }^{62}$ Cesium substitution introduces a compressive microstrain in the lattice from size mismatch $\left(r_{\mathrm{Cs}}=\right.$ $1.88 \AA ; r_{\mathrm{CH}\left(\mathrm{NH}_{2}\right)_{2}}=2.53 \AA$ ) that induces a local alignment of neighboring quadrupole moments, disrupting the " $\mathrm{T}$ " shape ordering and relieving geometric frustration (Figure 9e). ${ }^{64,65}$ Cesium 
substitution produces short-range ordered regions that interrupt the long-range concerted, changes in the reorientational dynamics at the crystallographically unresolvable phase transitions.

The relief of geometric frustration upon substitution contrasts with the behavior of $\mathrm{CH}_{3} \mathrm{NH}_{3}{ }^{+}$

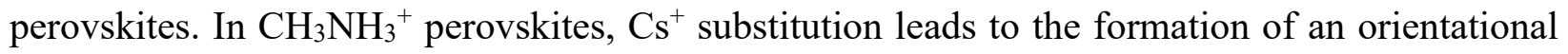
glass in the methylammonium sub-lattice with reduced $\mathrm{CH}_{3} \mathrm{NH}_{3}{ }^{+}$dynamics, which derives from the conflicting inorganic octahedral tilting preferences imparted by $\mathrm{CH}_{3} \mathrm{NH}_{3}{ }^{+}$vs $\mathrm{Cs}^{+} .{ }^{12}$ This contrasts with $\mathrm{CH}\left(\mathrm{NH}_{2}\right)_{2} \mathrm{PbBr}_{3}$ and $\mathrm{CsPBBr}_{3}$ which both display the same octahedral tilting distortions upon cooling. ${ }^{31,66}$ In the case of $\left(\mathrm{CH}\left(\mathrm{NH}_{2}\right)_{2}\right)_{1-x} \mathrm{Cs}_{x} \mathrm{PbBr}_{3}$, the retained local dynamics of the organic molecules prevents glass formation, but cesiation disrupts long-range ordered phase transitions. The INS data in particular demonstrate the presence of local microstrain-induced broadening upon substitution in $\left(\mathrm{CH}_{3} \mathrm{NH}_{3}\right)_{1-x} \mathrm{Cs}_{x} \mathrm{PbBr}_{3}$ and the comparative lack of local microstrain in $\left(\mathrm{CH}\left(\mathrm{NH}_{2}\right)_{2}\right)_{1-x} \mathrm{Cs}_{x} \mathrm{PbBr}_{3}$ (Figure 7), consistent with peak broadening observed in PXRD (Figure S5). The lack of disrupted vibrational modes at $T=5 \mathrm{~K}$ in $\left(\mathrm{CH}\left(\mathrm{NH}_{2}\right)_{2}\right)_{1-x} \mathrm{Cs}_{x} \mathrm{PbBr}_{3}$ with increasing cesium content emphasizes the high tolerance of hybrid perovskites towards substitution, as $\mathrm{Cs}^{+}$is nearly $70 \mathrm{pm}$ smaller than $\mathrm{CH}\left(\mathrm{NH}_{2}\right)_{2}{ }^{+}$. Each organic cation has markedly different responses upon substitution, which highlights the complexity of the potential energy landscape within mixed-cation perovskites commonly used in photovoltaic devices. ${ }^{5}$ The increased chemical stability of substituted formamidinium perovskite solar cells relative to unsubstituted solar cells ${ }^{67}$ could arise from the relief of geometric frustration from long-range elastic dipolar interactions upon $\mathrm{Cs}^{+}$substitution. 

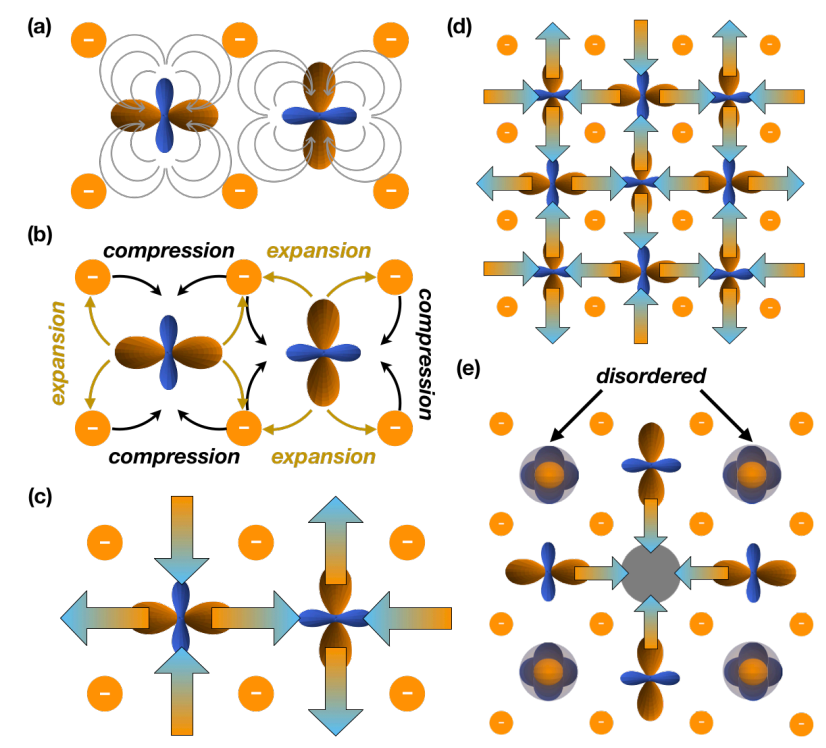

Figure 9: Schematic cartoon illustrating the role of quadrupolar and elastic interactions. (a) Electrostatic potential field lines emanating from the geometric representation of the quadrupolar moment tensor of formamidinium $^{60}$ (orange density denotes negative; blue is positive) provide attractive or repulsive interactions to the anionic structural framework, as depicted in (b). (c) The resulting expansive and compressive strain fields map onto an elastic dipole tensor model, depicted by the pairs of large arrows. (d) In a hypothetical two-dimensional plane, the elastic dipoles have a stable long-range ordered configuration that minimizes the elastic energy; however, a favorable three-dimensional configuration cannot be tiled. (e) Introduction of a smaller $\mathrm{Cs}^{+}$cation results in a neighboring compressive strain, thus causing a preferred orientation of quadrupolar cations around the $\mathrm{Cs}^{+}$and frustrated orientations of the next-nearest neighboring quadrupoles.

\section{Conclusions}

From ${ }^{1} \mathrm{H}$ NMR, ${ }^{14} \mathrm{~N}$ NMR, ${ }^{79} \mathrm{Br} \mathrm{NQR}$, and neutron scattering we confirm that the five phase transitions of $\mathrm{CH}\left(\mathrm{NH}_{2}\right)_{2} \mathrm{PbBr}_{3}$ are characterized by changes to the dynamic degrees of freedom of $\mathrm{CH}\left(\mathrm{NH}_{2}\right)_{2}{ }^{+}$, further corroborating previous studies. In particular, the ${ }^{14} \mathrm{~N}$ shows the progressive freezing of cation rotation, leaving only libration below $153 \mathrm{~K}$. Additionally, crystallography and $\mathrm{NQR}$ data show that the changes in dynamics couple to the inorganic octahedral framework through long-range cooperative tilting in the case of the crystallographically-resolved transitions, or local distortions of the bromine environment in the other phase transitions. The local compressive strain caused by Cs substitution overrides the orientation driven by the organicorganic interactions, disrupting the concerted changes at the low temperature phase transitions, which we have demonstrated through characterization of the series $\left(\mathrm{CH}\left(\mathrm{NH}_{2}\right)_{2}\right)_{1-x} \mathrm{Cs}_{x} \mathrm{PbBr}_{3}$. We propose that chemical substitution leads to the release of geometric frustration of $\mathrm{CH}\left(\mathrm{NH}_{2}\right)_{2}{ }^{+}$ interactions, while retaining the local dynamic degrees of freedom. This work provides insight into 
the sensitivity of the organic-organic interactions in $\mathrm{CH}\left(\mathrm{NH}_{2}\right)_{2} \mathrm{PbBr}_{3}$ and demonstrates that $\mathrm{Cs}^{+}$ substitution has important implications for the structure of hybrid perovskites that could explain the increased phase stability of chemically substituted hybrid perovskites that retain advantageous electronic properties.

\section{Experimental Methods}

Certain commercial equipment, instruments, or materials are identified in this paper in order to specify the experimental procedure adequately. This identification is not intended to imply recommendation or endorsement by the National Institute of Standards and Technology.

\section{Sample preparation:}

$\mathrm{CH}\left(\mathrm{NH}_{2}\right)_{2} \mathrm{PbBr}_{3}$ was prepared as previously described. ${ }^{30} \mathrm{In}$ brief, $\left[\mathrm{CH}\left(\mathrm{NH}_{2}\right)_{2}\right]\left[\mathrm{CH}_{3} \mathrm{COO}\right](\mathrm{VWR}$, 98\%) was dissolved in hydrobromic acid (Sigma Aldrich, 48\%) and reacted with $\mathrm{PbBr}_{2}(\mathrm{Sigma}$ Aldrich, $99.99 \%$ ) at $80{ }^{\circ} \mathrm{C}$. $\mathrm{CH}\left(\mathrm{NH}_{2}\right)_{2} \mathrm{PbBr}_{3}$ was precipitated with ethanol as an antisolvent and washed with ethanol. $\mathrm{CsPbBr}_{3}$ was prepared via a solid-state reaction from the binaries $\mathrm{CsBr}$ (VWR, 99.9\%) and $\mathrm{PbBr}_{2}$, carried out at $600{ }^{\circ} \mathrm{C}$ for six hours. The solid solutions were prepared by grinding molar quantities of the end members, sealing in an evacuated silica ampoule and gently heating at $150{ }^{\circ} \mathrm{C}$ for between one and three weeks.

\section{Diffraction:}

Laboratory powder X-ray diffraction (PXRD) data were collected on a Bruker D8 Discover X-ray diffractometer using a $\mathrm{Cu} \mathrm{K}_{\alpha}$ radiation source and a Lynxeye XE-T position-sensitive detector to check phase purity. Accurate lattice parameters were obtained using an internal silicon standard intimately mixed with each sample. High-resolution synchrotron powder X-ray diffraction (SXRD) patterns were collected on the diffractometer 11-BM-B at the Advanced Photon Source, Argonne National Laboratory $(\lambda \approx 0.41 \AA)$ between $T=300 \mathrm{~K}$ and $T=100 \mathrm{~K}$ in intervals of $\Delta \mathrm{T}=$ $10 \mathrm{~K} .{ }^{68}$ Powders were flame sealed in glass capillaries under vacuum.

\section{$N M R$ and $N Q R$ :}

Low-temperature ${ }^{1} \mathrm{H} T_{1}$ measurements $(<200 \mathrm{~K})$ were performed using the low-temperature magic angle spinning (MAS) capabilities at the UK DNP MAS NMR Facility at the University of Nottingham, on a 14.1 T AVANCE III HD spectrometer corresponding to a ${ }^{1} \mathrm{H}$ Larmor frequency 
of $600 \mathrm{MHz}$, with a $3.2 \mathrm{~mm}$ probe. All other NMR experiments were performed on a $16.4 \mathrm{~T}$ AVANCE III spectrometer (University of Cambridge) corresponding to a ${ }^{1} \mathrm{H}$ Larmor frequency of $700 \mathrm{MHz}$ using a $4 \mathrm{~mm}$ MAS probe. The NQR experiments were performed on an AVANCE spectrometer (University of Cambridge) using a $4 \mathrm{~mm}$ MAS probe designed for use with a $9.40 \mathrm{~T}$ (400 MHz) magnet.

The ${ }^{1} \mathrm{H} T_{1}$ constants were measured using a saturation recovery experiment with an appended Hahn echo, without (or with truncated) phase cycling to reduce the experimental time in the case of long $T_{1}$ constants.

${ }^{14} \mathrm{~N}$ NMR spectra in the cubic phase were measured at $5 \mathrm{kHz}$ MAS using a Hahn echo pulse sequence, a radiofrequency (rf) power of $41 \mathrm{kHz}$ and a recycle delay of $5 \mathrm{~ms}$; where the spectra were broader than could be excited in a single spectrum, variable offset cumulative spectroscopy (VOCS) acquisition was used in steps of $40 \mathrm{kHz}$. Below the cubic-tetragonal phase transition, ${ }^{14} \mathrm{~N}$ NMR spectra were recorded under static conditions with a WURST CPMG pulse sequence ${ }^{69-71}$ and an echo length of $200 \mu$ s yielding a spikelet separation of $5 \mathrm{kHz}$. Between 6 and 60 echoes were recorded depending on the $T_{2}$ constant, using $50 \mu \mathrm{s}$ WURST-80 pulses with a $500 \mathrm{kHz}$ excitation width and a rf power of $22 \mathrm{kHz}$; recycle delays of $5-50 \mathrm{~ms}$ were used, and continuous wave ${ }^{1} \mathrm{H}$ decoupling of $50 \mathrm{kHz}$ was applied. Probe ringing was removed by not recording the first two echoes, where the $T_{2}$ constant was sufficiently long to permit this. These ultra-wideline spectra were recorded using VOCS acquisition in steps of $100 \mathrm{kHz}$, with retuning achieved by an external automatic tuning and matching (eATM) robot, ${ }^{72}$ before being recombined by taking the magnitude data for each spectrum and performing a skyline projection. ${ }^{73}$ The symmetric $I=1$ quadrupolar interaction is expected to dominate the ${ }^{14} \mathrm{~N}$ spectrum, and so only the low-frequency half of the spectrum was recorded and then reflected to reproduce the complete spectrum.

${ }^{79} \mathrm{Br}$ NQR spectra were recorded with a Hahn echo pulse sequence, a rf power of $200 \mathrm{kHz}$, a recycle delay of $25 \mathrm{~ms}$ and VOCS acquisition in steps of $200 \mathrm{kHz}$, again with retuning by an eATM robot. The sample temperature for all experiments was determined with an ex situ temperature calibration using the temperature-dependent ${ }^{207} \mathrm{~Pb}$ chemical shift of $\mathrm{Pb}\left(\mathrm{NO}_{3}\right)_{2},{ }^{74}$ except for the lowtemperature ${ }^{1} \mathrm{H} T_{1}$ experiments $(<200 \mathrm{~K})$, for which the sample temperature was determined from an ex situ temperature calibration using the temperature-dependent ${ }^{79} \mathrm{Br} T_{1}$ constant of $\mathrm{KBr} .{ }^{56}{ }^{14} \mathrm{~N}$ $\mathrm{NMR}$ spectra were referenced to $\mathrm{NH}_{4} \mathrm{Cl}$ at $0 \mathrm{ppm}$. 
Inelastic Neutron Scattering (INS):

Data were collected using the indirect-geometry spectrometer VISION at the Spallation Neutron Source, Oak Ridge National Laboratory. The instrument measures neutron energy transfer in the range of -2 to $1000 \mathrm{meV}$, with a resolution of $\mathrm{dE} / \mathrm{E}=1.5 \%$. Powders were packed into aluminum canisters under a helium atmosphere. Data were collected between $T=5 \mathrm{~K}$ and $T=275 \mathrm{~K}$ in $25 \mathrm{~K}$ intervals, with data collection times up to 1.5 hours. Time-of-flight data were reduced in MANTID. ${ }^{75}$ Reduced data were modeled as damped harmonic oscillators in DAVE. ${ }^{76}$ To lend assistance to interpretation of the INS spectra, phonon calculations were performed, as discussed in the SI.

\section{Quasielastic Neutron Scattering (QENS):}

Fixed window elastic scattering and quasielastic spectroscopy experiments were performed on the high flux backscattering spectrometer (HFBS) at the NIST Center for Neutron Research on nondeuterated samples of $\mathrm{CH}\left(\mathrm{NH}_{2}\right)_{2} \mathrm{PbBr}_{3}{ }^{77}$ The sample environment employed in this experiment is a closed-cycle refrigerator that cools/warms the sample by helium exchange gas. A mass of 3.27 $\mathrm{g}$ of $\mathrm{CH}\left(\mathrm{NH}_{2}\right)_{2} \mathrm{PbBr}_{3}$ powder was used for this experiment. The cylindrical sample cell has a diameter of approximately $3 \mathrm{~cm}$, and the powder sample was held against the lateral area of the sample can by enclosing it within aluminum foil pouches. The sample cell was loaded within a helium glove box and sealed with an indium gasket. The enclosed helium ensures good thermal contact between the powder and the rest of the sample environment.

Fixed window scans were performed according to the following protocol. Initially, the sample was quenched by placing it within the well of the closed cycle refrigerator when it was at $60 \mathrm{~K}$. The sample temperature dropped rapidly to approximately $200 \mathrm{~K}$ and subsequently took $90 \mathrm{~min}$ to reach $6 \mathrm{~K}$. Data was continuously collected upon warming at $0.8 \mathrm{~K} / \mathrm{min}$ with $60 \mathrm{sec} /$ point. Finally, data was collected upon cooling at the same nominal ramp rate and time per point.

A fixed window scan is a measurement of the elastic intensity $I_{e l}(Q, T)$ as a function of $Q$ and temperature. The effective mean-squared displacement of the hydrogen atoms is estimated by assuming that $I_{e l}(Q, T)$ is governed by a Debye-Waller factor:

$I_{e l}(Q, T)=I_{e l}(Q, T=6.0 \mathrm{~K}) \times \exp \left(-Q^{2}\left\langle u^{2}\right\rangle / 3\right)$.

This calculation was performed using the DAVE software. ${ }^{76}$ 


\section{Acknowledgements}

This work was supported by the U.S. Department of Energy, Office of Science, Basic Energy Sciences, under Award SC0016083. J.R.N. and E.M.M acknowledge support from Research Corporation for Science Advancement through a Cottrell Scholar Award, and J.R.N. thanks the A.P. Sloan Foundation for assistance provided from a Sloan Research Fellowship. M.A.H. gratefully acknowledges an Oppenheimer studentship. D.M.H. acknowledges the Cambridge International Trust for funding, and is grateful for support from NECCES, an Energy Frontier Research Center funded by the U.S. Department of Energy, Office of Science, Office of Basic Energy Sciences under Award No. DE-SC0012583. We would like to thank Dr. Subhradip Paul, University of Nottingham, for assistance in recording the low-temperature ${ }^{1} \mathrm{H}$ NMR spectra. Access to the High Flux Backscattering Spectrometer was provided by the Center for High Resolution Neutron Scattering, a partnership between the National Institute of Standards and Technology and the National Science Foundation under Agreement No. DMR-1508249. A portion of this research used resources at the Spallation Neutron Source, a DOE Office of Science User

Facility operated by the Oak Ridge National Laboratory. Use of the Advanced Photon Source at Argonne National Laboratory was supported by the U.S. Department of Energy, Office of Science, Office of Basic Energy Sciences, under Contract No. DE-AC02-06CH11357.

\section{Supporting Information}

Additional details regarding DFT calculations, correlation times, diffraction data, neutron scattering and phonon calculations.

\section{Conflict of Interest}

The authors declare no competing interests.

\section{Table of Contents Figure}




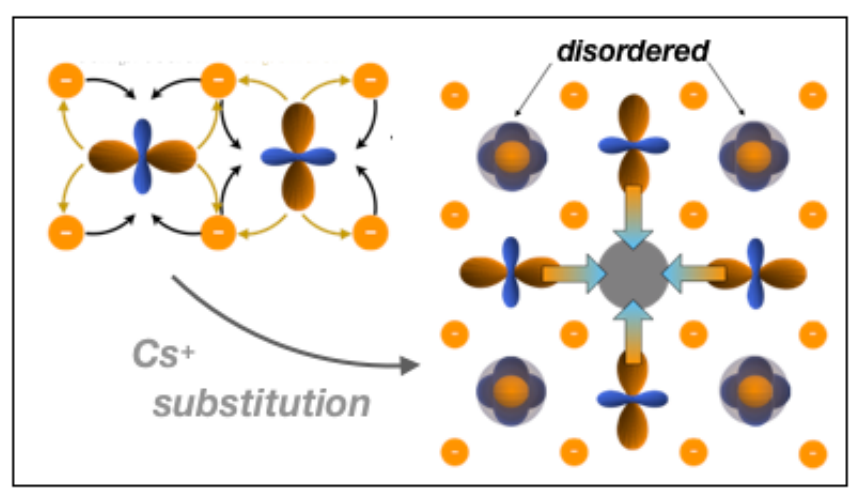




\section{References}

(1) Deretzis, I.; Smecca, E.; Mannino, G.; La Magna, A.; Miyasaka, T.; Alberti, A. Stability and Degradation in Hybrid Perovskites: Is the Glass Half-Empty or Half-Full? J. Phys. Chem. Lett. 2018, acs.jpclett.8b00120.

(2) Nagabhushana, G. P.; Shivaramaiah, R.; Navrotsky, A. Direct Calorimetric Verification of Thermodynamic Instability of Lead Halide Hybrid Perovskites. Proc. Natl. Acad. Sci. 2016, 113 (28), 7717-7721.

(3) Schelhas, L. T.; Li, Z.; Christians, J. A.; Goyal, A.; Kairys, P.; Harvey, S. P.; Kim, D. H.; Stone, K. H.; Luther, J. M.; Zhu, K.; et al. Insights into Operational Stability and Processing of Halide Perovskite Active Layers. Energy Environ. Sci. 2019, 12, 13411348.

(4) Chen, L.; Tan, Y.-Y.; Chen, Z.-X.; Wang, T.; Hu, S.; Nan, Z.-A.; Xie, L.-Q.; Hui, Y.; Huang, J.-X.; Zhan, C.; et al. Toward Long-Term Stability: Single-Crystal Alloys of Cesium-Containing Mixed Cation and Mixed Halide Perovskite. J. Am. Chem. Soc. 2019, jacs.8b11610.

(5) Christians, J. A.; Schulz, P.; Tinkham, J. S.; Schloemer, T. H.; Harvey, S. P.; Tremolet de Villers, B. J.; Sellinger, A.; Berry, J. J.; Luther, J. M. Tailored Interfaces of Unencapsulated Perovskite Solar Cells for \&gt;1,000 Hour Operational Stability. Nat. Energy 2018, 3 (1), 68-74.

(6) Li, Z.; Yang, M.; Park, J.-S. S.; Wei, S.-H. H.; Berry, J. J.; Zhu, K. Stabilizing Perovskite Structures by Tuning Tolerance Factor: Formation of Formamidinium and Cesium Lead Iodide Solid-State Alloys. Chem. Mater. 2016, 28 (1), 284-292.

(7) Lee, J. W.; Kim, D. H.; Kim, H. S.; Seo, S. W.; Cho, S. M.; Park, N. G. Formamidinium and Cesium Hybridization for Photo- and Moisture-Stable Perovskite Solar Cell. Adv. Energy Mater. 2015, 5 (20).

(8) Poorkazem, K.; Kelly, T. L. Compositional Engineering To Improve the Stability of Lead Halide Perovskites: A Comparative Study of Cationic and Anionic Dopants. ACS Appl. Energy Mater. 2018, 1 (1), 181-190.

(9) McMeekin, D. P.; Sadoughi, G.; Rehman, W.; Eperon, G. E.; Saliba, M.; Horantner, M. T.; Haghighirad, A.; Sakai, N.; Korte, L.; Rech, B.; et al. A Mixed-Cation Lead MixedHalide Perovskite Absorber for Tandem Solar Cells. Science (80-. ). 2016, 351 (6269), 
151-155.

(10) Prasanna, R.; Gold-Parker, A.; Leijtens, T.; Conings, B.; Babayigit, A.; Boyen, H.-G.;

Toney, M. F.; McGehee, M. D. Band Gap Tuning via Lattice Contraction and Octahedral

Tilting in Perovskite Materials for Photovoltaics. J. Am. Chem. Soc. 2017, 139 (32), 11117-11124.

(11) Selig, O.; Sadhanala, A.; Müller, C.; Lovrincic, R.; Chen, Z.; Rezus, Y. L. A.; Frost, J. M.; Jansen, T. L. C.; Bakulin, A. A. Organic Cation Rotation and Immobilisation in Pure and Mixed Methylammonium Lead-Halide Perovskites. J. Am. Chem. Soc. 2017, 139 (11), 4068-4074.

(12) Mozur, E. M.; Maughan, A. E.; Cheng, Y.; Huq, A.; Jalarvo, N.; Daemen, L. L.; Neilson, J. R. Orientational Glass Formation in Substituted Hybrid Perovskites. Chem. Mater. 2017, 29 (23), 10168-10177.

(13) Ferdani, D. W.; Pering, S. R.; Ghosh, D.; Kubiak, P.; Walker, A. B.; Lewis, S. E.; Johnson, A. L.; Baker, P. J.; Islam, M. S.; Cameron, P. J. Partial Cation Substitution Reduces Iodide Ion Transport in Lead Iodide Perovskite Solar Cells. Energy Environ. Sci. 2019, 12 (7), 2264-2272.

(14) Kulbak, M.; Cahen, D.; Hodes, G. How Important Is the Organic Part of Lead Halide Perovskite Photovoltaic Cells? Efficient CsPbBr 3 Cells. J. Phys. Chem. Lett. 2015, 6 (13), 2452-2456.

(15) Motta, C.; El-Mellouhi, F.; Kais, S.; Tabet, N.; Alharbi, F.; Sanvito, S. Revealing the Role of Organic Cations in Hybrid Halide Perovskite CH3NH3PbI3. Nat. Commun. 2015, 6 (1), 7026.

(16) Quarti, C.; Mosconi, E.; De Angelis, F. Structural and Electronic Properties of OrganoHalide Hybrid Perovskites from Ab Initio Molecular Dynamics. Phys. Chem. Chem. Phys. 2015, 17 (14), 9394-9409.

(17) Zhu, H.; Miyata, K.; Fu, Y.; Wang, J.; Joshi, P.; Niesner, D.; Williams, K. W.; Jin, S.; Zhu, X.-Y. Screening in Crystalline Liquids Protects Energetic Carriers in Hybrid Perovskites. Science 2016, 353 (6306), 1409-1413.

(18) Miyata, K.; Meggiolaro, D.; Tuan Trinh, M.; Joshi, P. P.; Mosconi, E.; Jones, S. C.; De Angelis, F.; Zhu, X. Y. Large Polarons in Lead Halide Perovskites. Sci. Adv. 2017, 3 (8), e1701217. 
(19) Hopper, T. R.; Gorodetsky, A.; Frost, J. M.; Müller, C.; Lovrincic, R.; Bakulin, A. A. Ultrafast Intraband Spectroscopy of Hot-Carrier Cooling in Lead-Halide Perovskites. ACS Energy Lett. 2018, 3 (9), 2199-2205.

(20) McKechnie, S.; Frost, J. M.; Pashov, D.; Azarhoosh, P.; Walsh, A.; van Schilfgaarde, M. Dynamic Symmetry Breaking and Spin Splitting in Metal Halide Perovskites. Phys. Rev. B 2018, 98 (8), 085108.

(21) Etienne, T.; Mosconi, E.; De Angelis, F. Dynamical Origin of the Rashba Effect in Organohalide Lead Perovskites: A Key to Suppressed Carrier Recombination in Perovskite Solar Cells? J. Phys. Chem. Lett. 2016, 7 (9), 1638-1645.

(22) Ghosh, D.; Smith, A. R.; Walker, A. B.; Islam, M. S. Mixed A-Cation Perovskites for Solar Cells: Atomic-Scale Insights into Structural Distortion, Hydrogen Bonding, and Electronic Properties. Chem. Mater. 2018, 30 (15), 5194-5204.

Onoda-Yamamuro, N.; Matsuo, T.; Suga, H. Calorimetric and IR Spectroscopic Studies of Phase Transitions in Methylammonium Trihalogenoplumbates (II). J. Phys. Chem. Solids 1990, 51 (12), 1383-1395.

(24) Ren, Y.; Oswald, I. W. H.; Wang, X.; McCandless, G. T.; Chan, J. Y. Orientation of Organic Cations in Hybrid Inorganic-Organic Perovskite CH3NH3PbI3 from Subatomic Resolution Single Crystal Neutron Diffraction Structural Studies. Cryst. Growth Des. 2016, 16 (5), 2945-2951.

(25) Fabini, D. H.; Siaw, T. A.; Stoumpos, C. C.; Laurita, G.; Olds, D.; Page, K.; Hu, J. G.; Kanatzidis, M. G.; Han, S.; Seshadri, R. Universal Dynamics of Molecular Reorientation in Hybrid Lead Iodide Perovskites. J. Am. Chem. Soc. 2017, 139 (46), 16875-16884.

(26) Maheshwari, S.; Fridriksson, M. B.; Seal, S.; Meyer, J.; Grozema, F. C. The Relation between Rotational Dynamics of the Organic Cation and Phase Transitions in Hybrid Halide Perovskites. J. Phys. Chem. C 2019, 123 (23), 14652-14661.

(27) Chen, T.; Foley, B. J.; Park, C.; Brown, C. M.; Harriger, L. W.; Lee, J.; Ruff, J.; Yoon, M.; Choi, J. J.; Lee, S.-H. Entropy-Driven Structural Transition and Kinetic Trapping in Formamidinium Lead Iodide Perovskite. Sci. Adv. 2016, 2 (10), e1601650.

(28) Fabini, D. H.; Stoumpos, C. C.; Laurita, G.; Kaltzoglou, A.; Kontos, A. G.; Falaras, P.; Kanatzidis, M. G.; Seshadri, R. Reentrant Structural and Optical Properties and Large Positive Thermal Expansion in Perovskite Formamidinium Lead Iodide. Angew. Chemie 
Int. Ed. 2016, 55 (49), 15392-15396.

(29) Keshavarz, M.; Ottesen, M.; Wiedmann, S.; Wharmby, M.; Küchler, R.; Yuan, H.;

Debroye, E.; Steele, J. A.; Martens, J.; Hussey, N. E.; et al. Tracking Structural Phase

Transitions in Lead-Halide Perovskites by Means of Thermal Expansion. Adv. Mater.

2019, 1900521, 1900521.

(30) Mozur, E. M.; Trowbridge, J. C.; Maughan, A. E.; Gorman, M. J.; Brown, C. M.; Prisk, T. R.; Neilson, J. R. Dynamical Phase Transitions and Cation Orientation-Dependent Photoconductivity in $\mathrm{CH}(\mathrm{NH} 2) 2 \mathrm{PbBr3}$. ACS Mater. Lett. 2019, 1 (2), 260-264.

(31) Schueller, E. C.; Laurita, G.; Fabini, D. H.; Stoumpos, C. C.; Kanatzidis, M. G.; Seshadri, R. Crystal Structure Evolution and Notable Thermal Expansion in Hybrid Perovskites Formamidinium Tin Iodide and Formamidinium Lead Bromide. Inorg. Chem. 2018, 57 (2), 695-701.

(32) Rosales, B. A.; Hanrahan, M. P.; Boote, B. W.; Rossini, A. J.; Smith, E. A.; Vela, J. Lead Halide Perovskites: Challenges and Opportunities in Advanced Synthesis and Spectroscopy. ACS Energy Lett. 2017, 2 (4), 906-914.

(33) Franssen, W. M. J.; Kentgens, A. P. M. Solid-State NMR of Hybrid Halide Perovskites. Solid State Nucl. Magn. Reson. 2019, 100, 36-44.

(34) Roiland, C.; Trippé-Allard, G.; Jemli, K.; Alonso, B.; Ameline, J.-C.; Gautier, R.; Bataille, T.; Le Pollès, L.; Deleporte, E.; Even, J.; et al. Multinuclear NMR as a Tool for Studying Local Order and Dynamics in $\mathrm{CH} 3 \mathrm{NH} 3 \mathrm{PbX} 3(\mathrm{X}=\mathrm{Cl}, \mathrm{Br}, \mathrm{I})$ Hybrid Perovskites. Phys. Chem. Chem. Phys. 2016, 18 (27), 18112-18118.

(35) Askar, A. M.; Bernard, G. M.; Wiltshire, B.; Shankar, K.; Michaelis, V. K. Multinuclear Magnetic Resonance Tracking of Hydro, Thermal, and Hydrothermal Decomposition of CH3NH3PbI3. J. Phys. Chem. C 2017, 121 (2), 1013-1024.

(36) Wasylishen, R. E.; Knop, O.; Macdonald, J. B. Cation Rotation in Methylammonium Lead Halides. Solid State Commun. 1985, 56 (7), 581-582.

(37) Kubicki, D. J.; Prochowicz, D.; Hofstetter, A.; Péchy, P.; Zakeeruddin, S. M.; Grätzel, M.; Emsley, L.; Pechy, P.; Zakeeruddin, S. M.; Grätzel, M.; et al. Cation Dynamics in MixedCation (MA) x (FA) 1-x PbI 3 Hybrid Perovskites from Solid-State NMR. J. Am. Chem. Soc. 2017, 139 (29), 10055-10061.

(38) Xu, Q.; Eguchi, T.; Nakayama, H.; Nakamura, N.; Kishita, M. Molecular Motions and 
Phase Transitions in Solid CH3NH3PbX3 $(\mathrm{X}=\mathrm{Cl}, \mathrm{Br}, \mathrm{I})$ as Studied by NMR and NQR. Z. Naturforsch 1991, 46a, 240-246.

(39) Volkov, A. F.; Venevtsev, Y. N.; Semin, G. K. Nuclear Quadrupole Resonance (NQR) of Bromine-79 and Bromine-81 [and Iodine-127] in Perovskite and Orthorhombic Forms of CsPbBr3 and CsPbI3. Phys. Status Solidi 1969, 35, K167-K169.

(40) Bernard, G. M.; Wasylishen, R. E.; Ratcliffe, C. I.; Terskikh, V.; Wu, Q.; Buriak, J. M.; Hauger, T. Methylammonium Cation Dynamics in Methylammonium Lead Halide Perovskites: A Solid-State NMR Perspective. J. Phys. Chem. A 2018, 122 (6), 1560-1573.

(41) Senocrate, A.; Moudrakovski, I.; Maier, J. Short-Range Ion Dynamics in Methylammonium Lead Iodide by Multinuclear Solid State NMR and 127I NQR. Phys. Chem. Chem. Phys. 2018, 20 (30), 20043-20055.

(42) Widdifield, C. M.; Chapman, R. P.; Bryce, D. L. Chlorine, Bromine, and Iodine SolidState NMR Spectroscopy in Annual Reports on NMR Spectroscopy; Lindman, B., Forsen, S., Eds.; Elsevier Ltd.: Berlin, 2009; Vol. 66.

(43) Szell, P. M. J.; Bryce, D. L. Recent Advances in Chlorine, Bromine, and Iodine SolidState NMR Spectroscopy in Annual Reports on NMR Spectroscopy, 1st ed.; Webb, G. A., Ed.; Elsevier Ltd.: London, 2015; Vol. 84.

(44) Li, B.; Kawakita, Y.; Liu, Y.; Matsuura, M.; Shibata, K.; Kawamura, S.; Yamada, T.; Nakajima, K.; Shengzhong; Liu. Rotor-Phonon Coupling in Perovskite CH3NH3PbI3: The Origin of Exceptional Transport Properties. 2016, arxiv:1612.01631.

(45) Swainson, I. P.; Stock, C.; Parker, S. F.; Van Eijck, L.; Russina, M.; Taylor, J. W. From Soft Harmonic Phonons to Fast Relaxational Dynamics in $\mathrm{CH}$ _3 $\$ \mathrm{NH}$ \&3\$PbBr\$3\$. Phys. Rev. B 2015, 92 (10), 100303.

(46) Bechtel, J. S.; Seshadri, R.; Van der Ven, A. Energy Landscape of Molecular Motion in Cubic Methylammonium Lead Iodide from First-Principles. J. Phys. Chem. C 2016, 120 (23), 12403-12410.

(47) Bloembergen, N.; Purcell, E. M.; Pound, R. V. Relaxation Effects in Nuclear Magnetic Resonance Absorption. Phys. Rev. 1948, 73 (7), 679-712.

(48) Jeffrey, K. R.; Penner, G. H. Structural Phase Transitions. In NMR Crystallography; Harris, R. K., Wasylishen, R. E., Duer, M. J., Eds.; Wiley: Chichester, 2009; pp 387-413.

Page, K.; Siewenie, J. E.; Quadrelli, P.; Malavasi, L. Short-Range Order of 
Methylammonium and Persistence of Distortion at the Local Scale in MAPbBr 3 Hybrid Perovskite. Angew. Chemie Int. Ed. 2016, 55 (46), 14320-14324.

(50) Farid, U.; Khan, H. U.; Avdeev, M.; Injac, S.; Kennedy, B. J. Structural Studies of the High Temperature Phases of AgTaO3. J. Solid State Chem. 2018, 258 (December 2017), 859-864.

(51) Howard, C. J.; Knight, K. S.; Kennedy, B. J.; Kisi, E. H. Structural Phase Transitions in Strontium Zirconate Revisited. J. Phys. Condens. Matter 2000, 12 (45).

(52) Brivio, F.; Frost, J. M.; Skelton, J. M.; Jackson, A. J.; Weber, O. J.; Weller, M. T.; Goñi, A. R.; Leguy, A. M. A.; Barnes, P. R. F.; Walsh, A. Lattice Dynamics and Vibrational Spectra of the Orthorhombic, Tetragonal, and Cubic Phases of Methylammonium Lead Iodide. Phys. Rev. B - Condens. Matter Mater. Phys. 2015, 92 (144308), 1-8.

(53) Ivanovska, T.; Quarti, C.; Grancini, G.; Petrozza, A.; De Angelis, F.; Milani, A.; Ruani, G. Vibrational Response of Methylammonium Lead Iodide: From Cation Dynamics to Phonon-Phonon Interactions. ChemSusChem 2016, 1-12.

(54) Leguy, A. M. A.; Goñi, A. R.; Frost, J. M.; Skelton, J.; Brivio, F.; Rodríguez-martínez, X.; Weber, O. J.; Pallipurath, A. Dynamic Disorder, Phonon Lifetimes, and the Assignment of Modes to the Vibrational Spectra of Methylammonium Lead Halide Perovskites. Phys. Chem. Chem. Phys. 2016, 18, 27051-27066.

(55) Wilkening, M.; Heitjans, P. From Micro to Macro: Access to Long-Range Li+ Diffusion Parameters in Solids via Microscopic 6, 7Li Spin-Alignment Echo NMR Spectroscopy. ChemPhysChem 2012, 13 (1), 53-65.

(56) Thurber, K. R.; Tycko, R. Measurement of Sample Temperatures under Magic-Angle Spinning from the Chemical Shift and Spin-Lattice Relaxation Rate of $79 \mathrm{Br}$ in $\mathrm{KBr}$ Powder. J. Magn. Reson. 2009, 196 (1), 84-87.

(57) Fabini, D. H.; Hogan, T.; Evans, H. A.; Stoumpos, C. C.; Kanatzidis, M. G.; Seshadri, R. Dielectric and Thermodynamic Signatures of Low-Temperature Glassy Dynamics in the Hybrid Perovskites CH3NH3PbI3 and HC (NH2) 2PbI3. J. Phys. Chem. Lett. 2016, 7 (3), $376-381$.

(58) Young, J.; Rondinelli, J. M. Octahedral Rotation Preferences in Perovskite Iodides and Bromides. J. Phys. Chem. Lett. 2016, 7 (5), 918-922.

(59) Lee, J.-H.; Bristowe, N. C.; Lee, J. H.; Lee, S.-H.; Bristowe, P. D.; Cheetham, A. K.; Jang, 
H. M. Resolving the Physical Origin of Octahedral Tilting in Halide Perovskites. Chem. Mater. 2016, 28 (12), 4259-4266.

(60) Chen, T.; Chen, W.-L.; Foley, B. J.; Lee, J.; Ruff, J. P. C.; Ko, J. Y. P.; Brown, C. M.; Harriger, L. W.; Zhang, D.; Park, C.; et al. Origin of Long Lifetime of Band-Edge Charge Carriers in Organic-Inorganic Lead Iodide Perovskites. Proc. Natl. Acad. Sci. 2017, 114 (29), 7519-7524.

(61) Fredrickson, D. C. DFT-Chemical Pressure Analysis: Visualizing the Role of Atomic Size in Shaping the Structures of Inorganic Materials. J. Am. Chem. Soc. 2012, 134 (13), 59915999.

(62) Grannan, E. R.; Randeria, M.; Sethna, J. P. Low-Temperature Properties of a Model Glass. I. Elastic Dipole Model. Phys. Rev. B. 1990, 41 (11), 7784-7799.

(63) Ferreira, A. C.; Létoublon, A.; Paofai, S.; Raymond, S.; Ecolivet, C.; Rufflé, B.; Cordier, S.; Katan, C.; Saidaminov, M. I.; Zhumekenov, A. A.; et al. Elastic Softness of Hybrid Lead Halide Perovskites. Phys. Rev. Lett. 2018, 121 (8), 085502.

(64) Shannon, R. D.; Prewitt, C. T. Effective Ionic Radii in Oxides and Fluorides. Acta Crystallogr. Sect. B Struct. Crystallogr. Cryst. Chem. 1969, 25 (5), 925-946.

(65) Kieslich, G.; Sun, S.; Cheetham, T. An Extended Tolerance Factor Approach for OrganicInorganic Perovskites. Chem. Sci. 2015, 6 (6), 3430-3433.

(66) Rodova, M.; Brozek, J.; Knizek, K.; Nitsch, K. Phase Transitions in Ternary Caesium Lead Bromide. J. Therm. Anal. Calorim. 2003, 71 (2), 667-673.

(67) Bush, K. A.; Palmstrom, A. F.; Yu, Z. J.; Boccard, M.; Cheacharoen, R.; Mailoa, J. P.; McMeekin, D. P.; Hoye, R. L. Z.; Bailie, C. D.; Leijtens, T.; et al. 23.6\%-Efficient Monolithic Perovskite/Silicon Tandem Solar Cells with Improved Stability. Nat. Energy 2017, 2 (4), 17009.

(68) Wang, J.; Toby, B. H.; Lee, P. L.; Ribaud, L.; Antao, S. M.; Kurtz, C.; Ramanathan, M.; Von Dreele, R. B.; Beno, M. A. A Dedicated Powder Diffraction Beamline at the Advanced Photon Source: Commissioning and Early Operational Results. Rev. Sci. Instrum. 2008, 79 (8), 1-7.

(69) Kupče, E.; Freeman, R. Adiabatic Pulses for Wideband Inversion and Broadband Decoupling. J. Magn. Reson. Ser. A 1995, 273-276.

(70) Veinberg, S. L.; Lindquist, A. W.; Jaroszewicz, M. J.; Schurko, R. W. Practical 
Considerations for the Acquisition of Ultra-Wideline 14N NMR Spectra. Solid State Nucl. Magn. Reson 2017, 45-58.

(71) O’Dell, L. A. 14N Solid-State NMR. In Modern Methods in Solid-state NMR: A Practitioner's Guide; Hodgkinson, P., Ed.; The Royal Society of Chemistry: London, 2018.

(72) Pecher, O.; Halat, D. M.; Lee, J.; Liu, Z.; Griffith, K. J.; Braun, M.; Grey, C. P. Enhanced Efficiency of Solid-State NMR Investigations of Energy Materials Using an External Automatic Tuning/Matching (EATM) Robot. J. Magn. Reson 2017, 275, 127-136.

(73) O'Dell, L. A. ${ }^{14} \mathrm{~N}$ Solid-State NMR. In Modern Methods in Solid-state NMR: A Practitioner's Guide; Hodgkinson, P., Ed.; The Royal Society of Chemistry: London, 2018.

(74) Bielecki, A.; Burum, D. P. Temperature Dependence of 207Pb MAS Spectra of Solid Lead Nitrate. An Accurate, Sensitive Thermometer for Variable-Temperature MAS. $J$. Magn. Reson. Ser. A. 1995, 116, 215-220.

(75) Arnold, O.; Bilheux, J. C.; Borreguero, J. M.; Buts, A.; Campbell, S. I.; Chapon, L.; Doucet, M.; Draper, N.; Ferraz Leal, R.; Gigg, M. A.; et al. Mantid - Data Analysis and Visualization Package for Neutron Scattering and $\mu$ SR Experiments. Nucl. Instruments Methods Phys. Res. Sect. A Accel. Spectrometers, Detect. Assoc. Equip. 2014, 764, 156166.

(76) Azuah, R. T.; Kneller, L. R.; Qiu, Y.; Tregenna-Piggott, P. L. W.; Brown, C. M.; Copley, J. R. D.; Dimeo, R. M. DAVE: A Comprehensive Software Suite for the Reduction, Visualization, and Analysis of Low Energy Neutron Spectroscopic Data. J. Res. Natl. Inst. Stan. Technol. 2009, 114, 341-358.

(77) Meyer, A.; Dimeo, R. M.; Gehring, P. M.; Neumann, D. A. The High-Flux Backscattering Spectrometer at the NIST Center for Neutron Research. Rev. Sci. Instrum. 2003, 74 (5), 2759-2777. 